\title{
DLR project Digital-X: towards virtual aircraft design and flight testing based on high-fidelity methods
}

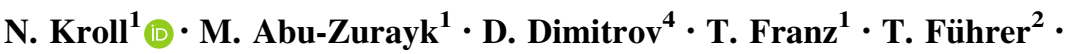

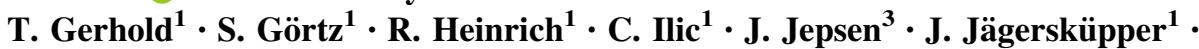 \\ M. Kruse ${ }^{1}$ A. Krumbein ${ }^{1}$ S. Langer ${ }^{1}$ D. Liu ${ }^{1} \cdot$ R. Liepelt ${ }^{4} \cdot$ L. Reimer $^{1}$.

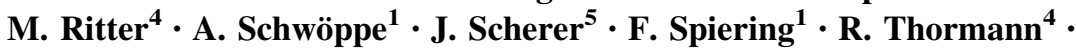 \\ V. Togiti ${ }^{1} \cdot$ D. Vollmer ${ }^{1} \cdot$ J.-H. Wendisch ${ }^{1}$
}

Received: 1 May 2015/Revised: 18 September 2015/Accepted: 19 November 2015/Published online: 22 December 2015

(C) The Author(s) 2015. This article is published with open access at Springerlink.com

\begin{abstract}
Numerical simulation is already an important cornerstone for aircraft design, although the application of highly accurate methods is mainly limited to the design point. To meet future technical, economic and social challenges in aviation, it is essential to simulate a real aircraft at an early stage, including all multidisciplinary interactions covering the entire flight envelope, and to have the ability to provide data with guaranteed accuracy required for development and certification. However, despite the considerable progress made there are still significant obstacles to be overcome in the development of numerical methods, physical modeling, and the integration of different aircraft disciplines for multidisciplinary analysis and optimization of realistic aircraft configurations. At DLR, these challenges are being addressed in the framework of the multidisciplinary project Digital-X (4/ 2012-12/2015). This paper provides an overview of the project objectives and presents first results on enhanced
\end{abstract}

This paper is based on a presentation at the German Aerospace Congress, September 16-18, 2014, Augsburg, Germany.

N. Kroll

norbert.kroll@dlr.de

1 Institute of Aerodynamics and Flow Technology, German Aerospace Center (DLR), Lilienthalplatz 7,

38108 Braunschweig, Germany

2 Institute of Composite Structures and Adaptive Systems, German Aerospace Center (DLR), Braunschweig, Germany

3 Institute of Air Transportation Systems, German Aerospace Center (DLR), Hamburg, Germany

4 Institute of Aeroelasticity, German Aerospace Center (DLR), Göttingen, Germany

5 Institute of Structures and Design, German Aerospace Center (DLR), Stuttgart, Germany disciplinary methods in aerodynamics and structural analysis, the development of efficient reduced order methods for load analysis, the development of a multidisciplinary optimization process based on a multi-level/variable-fidelity approach, as well as the development and application of multidisciplinary methods for the analysis of maneuver loads.

Keywords Virtual aircraft · High-fidelity methods . Multidisciplinary optimization

\section{Introduction}

In recent years, the aeronautical industry has established numerical flow simulations as a key element in the aerodynamic design process, complementing wind tunnel and flight tests. The continuous development of physical models and numerical methods and the availability of increasingly powerful computers suggest using numerical simulations to a much greater extent than in the past, radically changing the way aircraft will be designed in the future. In addition to speeding up and improving the product design cycle, numerical simulation also provides the possibility to mathematically model all properties of the designed product with their interactions and to determine the behavior under realistic operating conditions. With suitable high-fidelity multidisciplinary simulation methods at hand, the flight characteristics of an aircraft can be determined through numerical computation and the flight envelope can be flown virtually before the real first flight is performed. The realization of the vision of an aircraft performing its maiden flight in a virtual computer environment, denoted here by the synonym Digital-X, offers the reduction of development risks and in the medium and 
long-term significant cuts in development costs through stepwise certification.

In the context of the vast future challenges for the aircraft industry (Green aircraft, [1]), numerical simulation is considered to be a key technology for development of new or improvement of existing aircraft configurations. Thus, development and industrialization of advanced simulation methods and processes are being highly prioritized worldwide ([2-5]). At DLR, the multidisciplinary project Digital-X (04/2012-12/2015) represents a first significant building block for the progressive realization of the vision of digital aircraft design and virtual flight testing.

In this overview paper the main objectives of the project are explained in details and first results are presented from the various areas of work.

\section{Objectives and project set-up}

The primary objective of the project Digital-X is the development and deployment of a flexible, parallel software platform for multidisciplinary analysis and optimization of aircraft and helicopters based on high-fidelity numerical methods for each discipline involved. This platform will provide a robust, integrated design process for aerodynamics and structural analysis. This will break up the predominantly sequential approach currently used in detail design and the full potential of multidisciplinary design will be made available. This new software platform will also make it possible to efficiently and reliably perform maneuver simulations throughout the entire flight envelope, and thus permit the determination of aerodynamic and aeroelastic data for evaluating the handling qualities based on high-fidelity numerical methods. The simulation capabilities of the platform will be demonstrated through application-oriented design tasks and maneuver scenarios. This multidisciplinary simulation and optimization system will enable DLR to evaluate innovative technologies for new aircraft configurations based on high-fidelity methods. Due to the multidisciplinary objectives of Digital-X several institutes of DLR are involved and they contribute their specific expertise in a wide range of disciplines. Participants are the Institute of Aerodynamics and Flow Technology, Institute of Aeroelasticity, Institute of Propulsion Technology, Institute of Structures and Design, Institute of Composite Structures and Adaptive Systems, Institute of Flight Systems, Institute of Air Transportation Systems, Institute of System Dynamics and Control and DLR Simulation and Software Technology. The overall coordination is carried out by the Institute of Aerodynamics and Flow Technology.

The project runs for 4 years and is scheduled to end in June 2016. Seven research fields are addressed as indicated in Fig. 1. Note that the methods and tools for structural analysis are further developed in the work package dealing with multidisciplinary optimization, while there is a dedicated work package for the development of aerodynamic methods.

In the frame of the Digital-X project DLR works in partnership with aircraft industry and selected universities. The associated partnership with Airbus allows on the one hand the consideration of operating conditions relevant to industrial applications of numerical simulation methods and processes at an early stage and, on the other hand, joint validation activities taking into account industrial experience and data sets. It was agreed that the Airbus large transport aircraft of the eXternal Research Forum (XRF), the XRF-1, is used as a reference configuration for the developments in Digital-X. For this purpose global aircraft data, CAD geometry and structural models are being provided by Airbus. Specific knowledge of German universities in physical modeling as well as simulation and process chain developments are incorporated through a close link with collaborative research projects funded within the frame of the fourth aviation program of the German Federal Government.

Fig. 1 Main research fields of Digital-X

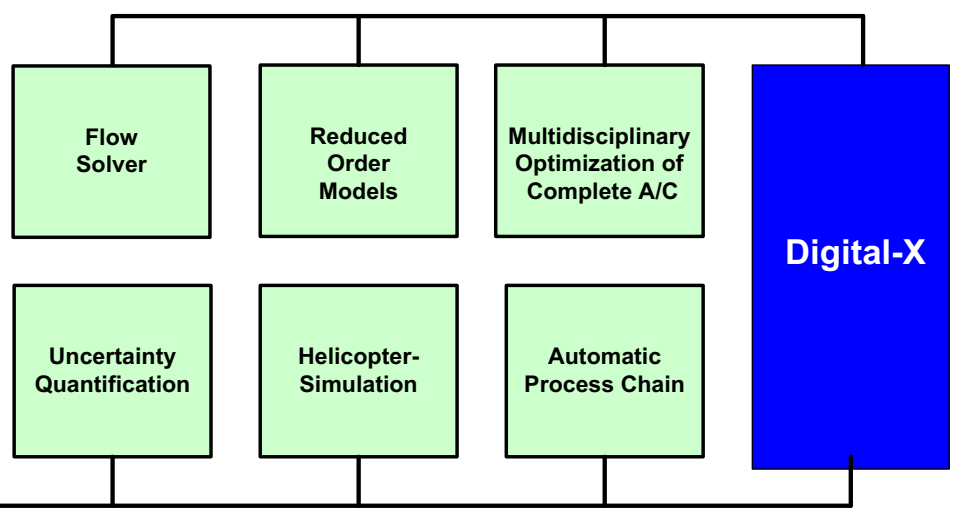




\section{Current work and results}

\subsection{Flow solver}

The unstructured DLR flow solver TAU routinely used in industry and research for aerodynamic applications is being further developed and improved in Digital-X for the required multidisciplinary flow simulations over the entire flight envelope. Furthermore, the development of the DLR 'next generation' CFD solver is being driven forward. In addition to consolidating existing methods and algorithms it will use innovative simulation approaches and provide the best possible utilization of future HPC systems.

\subsubsection{Further development of the DLR TAU code}

The key aspects of further developments in the hybrid TAU code [6] are to improve physical modeling and to increase both robustness and efficiency of the solver algorithms.

3.1.1.1 Physical modeling Several lines of development in the area of modeling are being followed in Digital-X. One is to target improvements in RANS turbulence models, which in practice are the basis of most of the turbulent simulations. The focus is on the correct capture of moderate separated flows on curved surfaces which cause separation at wings and determine the size and angle of attack of maximum lift. Particular features available in the DLR TAU code are the Reynolds Stress Models (RSM) [79]. They represent the highest level of RANS modeling and describe essential physical phenomena, which cannot be predicted by classical turbulent viscosity models used in industry. In Fig. 2 computed polars for the wing/fuselage configuration of the NASA Common Research Model (CRM) are compared with experimental data [10]. Note,

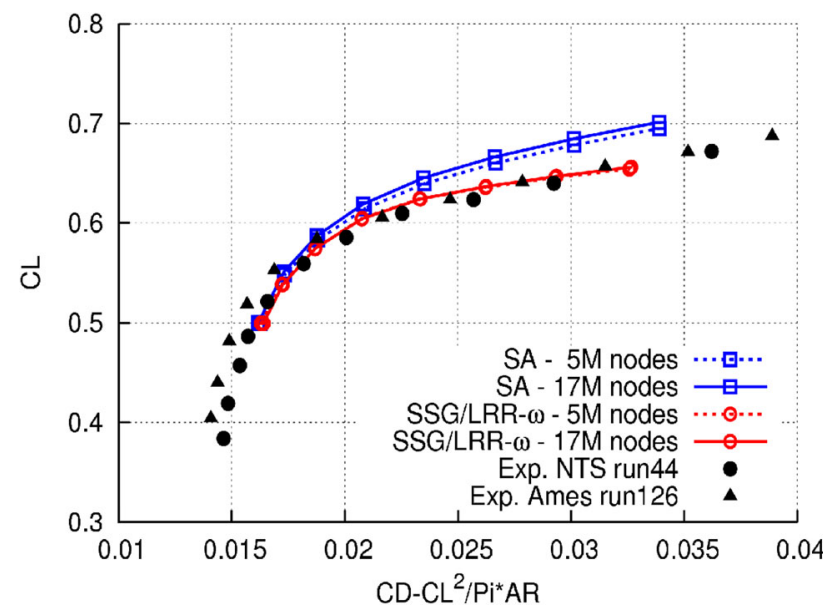

Fig. 2 Comparison of polars for the CRM wing/fuselage configuration $\left(M=0.85, R e=5 \times 10^{6}\right)$ that lift is plotted in terms of idealized profile drag where the estimate of induced drag is subtracted from the drag coefficient, enabling the use of expanded drag scales. The figure shows that the Reynolds stress model results (SSG/ LLR) clearly agree better with experimental data than results obtained with the single equation turbulence model of Spalart-Allmaras (SA). There is also a much lower dependency on mesh refinement. Efforts are currently being made to improve the numerical stability of the standard RSM in the TAU code for complex applications to ensure routine use under industrial conditions.

Techniques for scale resolving simulations (hybrid RANS/LES) have been expanded and improved as required to capture turbulent fluctuations in highly non-stationary processes, for example in the region downstream of components and attachments [11]. In particular for modeling weak flow separation on curved surfaces, a DES-based approach was developed using the thickness of the adjacent turbulent boundary layer and the separation point in the model to separate RANS and LES regions from each other as accurately as possible. Furthermore, the discretization scheme was adjusted so that LES results could be obtained with better quality.

For the simulation of transitional flows the range of applications for the automatic transition prediction was extended, which in its basic concept uses an $\mathrm{e}^{\mathrm{N}}$-method. As an alternative option a correlation-based transport equation transition model was implemented [12] and extended to the simulation of cross-flow instabilities [13]. Another line of development recently started was to couple this approach and the hybrid RANS/LES methods with the Reynolds stress models.

3.1.1.2 Improvements in efficiency and robustness Implicit solution methods are superior to explicit algorithms through their increased robustness, which is accompanied by additional cost particularly in memory and CPU time. The implicit solver currently available in the DLR TAU code is based on the LU-SGS algorithm. This procedure is characterized by a comparatively low memory and computational effort, at the expense of significant simplifications in the model derivation and a simple iterative solution method to solve the associated linear system of equations. However, due to increased computing capacity a loss in both robustness and efficiency is frequently found with the LU-SGS method for current applications with increasing size and complexity.

Digital-X activities are focused on improvements to the LU-SGS method as a smoother for agglomerated multi-grid techniques and on the further development of implicit methods, which both improve the approximations in the derivatives and also integrate efficient solution methods for the resulting equations into the overall process [14]. The 
single equation turbulence model of Spalart and Allmaras was initially applied to improve the solver algorithms. Recommendations from [15] were incorporated in the TAU code and the LU-SGS adapted accordingly. For example the limit of the transport variable of the turbulence model was removed from the TAU code. In addition, within the multigrid framework the smoothing properties of the LU-SGS in combination with various agglomeration techniques were investigated. It has been shown that the use of agglomeration techniques exploring specific features of structured grid regions in the boundary layer is advantageous. Furthermore, appropriate boundary conditions were implemented in the correction terms in the multi-grid and several smoothing steps were realized at each multi-grid level. These adjustments resulted in a significant increase in robustness and efficiency of the LU-SGS method in the TAU code.

As mentioned above improved preconditioning techniques were developed for implicit methods. These techniques are still based on the derivative computed by nearest neighbor information, but all derivative terms are consistently incorporated in the Jacobian matrix [16]. Furthermore, the symmetrical Gauss-Seidel algorithm is not terminated by a single iteration step, but several iterations are carried out to obtain better approximations to the solutions of the linear system of equations. To improve the convergence behavior for strongly anisotropic meshes, line iterative solution methods are applied.

The CRM wing/fuselage configuration from the 5th AIAA Drag Prediction Workshop was analyzed to demonstrate the algorithms developed [14]. A sequence of meshes was considered (Table 1) to assess the convergence behavior of the various methods. A reduction in residuals of density and of the turbulence equation was achieved up to machine accuracy for all meshes considered (Fig. 3). A CFL number of 1000 could be used for the newly implemented implicit method for all coarse meshes, although the achievable CFL number for the LU-SGS method varies between 2.5 and 25 , depending on the mesh. On the finest mesh the CFL number of the newly implemented implicit method had to be reduced to 250 .

The high CFL number demonstrates the increase in robustness for implicit methods. However, given the current state of development, a final conclusion could not be made regarding the efficiency of the newly integrated

Table 1 Mesh density for CRM wing/body-configuration

\begin{tabular}{lcrr}
\hline Level & No. of prisms & No. of tetrahedra & No. of points \\
\hline L1 & 425,984 & 255,904 & 660,177 \\
L2 & $1,437,696$ & $8,626,176$ & $2,204,089$ \\
L3 & $3,301,376$ & $20,766,720$ & $5,196,193$ \\
L4 & $11,261,952$ & $69,728,256$ & $17,441,905$ \\
L5 & $26,411,008$ & $166,133,760$ & $41,231,169$ \\
\hline
\end{tabular}

implicit method in the TAU code. This is the subject of further work.

\subsubsection{Next generation CFD solver}

This activity is concerned with the design and implementation of a flow solver of the next generation solver Flucs. The modular design of this software is constructed so that it can be used in highly parallel simulations for complex applications in the field of internal and external flows. The aim is to provide the basis for a consolidated flow solver using modern software techniques with high flexibility and high degree of innovation for a wide range of applications. This should make it possible to carry over the established models and methods of the DLR TAU code and combine them with new, innovative numerical approaches. An important aspect in developing the next generation solver Flucs is the efficient use of current and future parallel HPC systems.

Detailed specifications for Flucs were established based on an extensive survey of current and potential users of flow solvers in industrial and scientific fields. In addition to users, developers of current flow solvers were questioned to be able to make meaningful design decisions for the basic structure in many extension scenarios and thereby achieve high flexibility. After prioritization of the requirements, which implicitly defines a framework for the scope of work in Digital-X, a requirements specification was developed with concrete working points for implementation in a prototype. The focus here was on a common framework for two typical discretizations: second order finite volume and discontinuous Galerkin with variable order.

Not only future extensibility towards foreseeable developments, but also novel approaches are a core aspect of Flucs, but at the same time difficult to plan and control. Therefore, the development of a monolithic "all-embracing" flow solver was rejected, since the broad requirements cause potentially complex interactions and dependencies in various functions. Instead a "framework" (Fig. 4) was designed, whose data structures and functions serve as a basis for implementing lean modules, for example equations, discretizations and time integration procedures. The framework takes over many functions such as efficient implementation of loops, parallelization, support for algorithmic differentiation (AD) or the provision of required data.

The highest control module is designed as compatible Python API for the simulation environment FlowSimulator (see Sect. 3.7.1) which can flexibly describe a wide variety of simulation scenarios, since flow solver applications take place increasingly in a multidisciplinary environment. $\mathrm{C}++11$ was chosen as implementation language, which provides an object-oriented design (flexibility and modularity), together with a "close to the hardware" implementation of run-time critical program parts when required. 
(a)

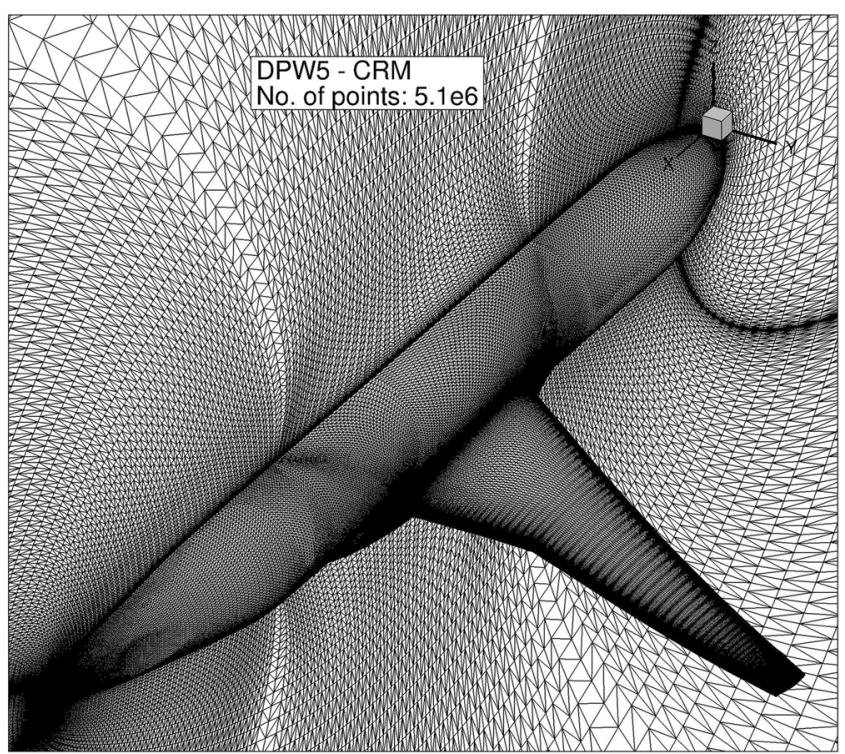

(c) DPW5 CRM, Implicit method

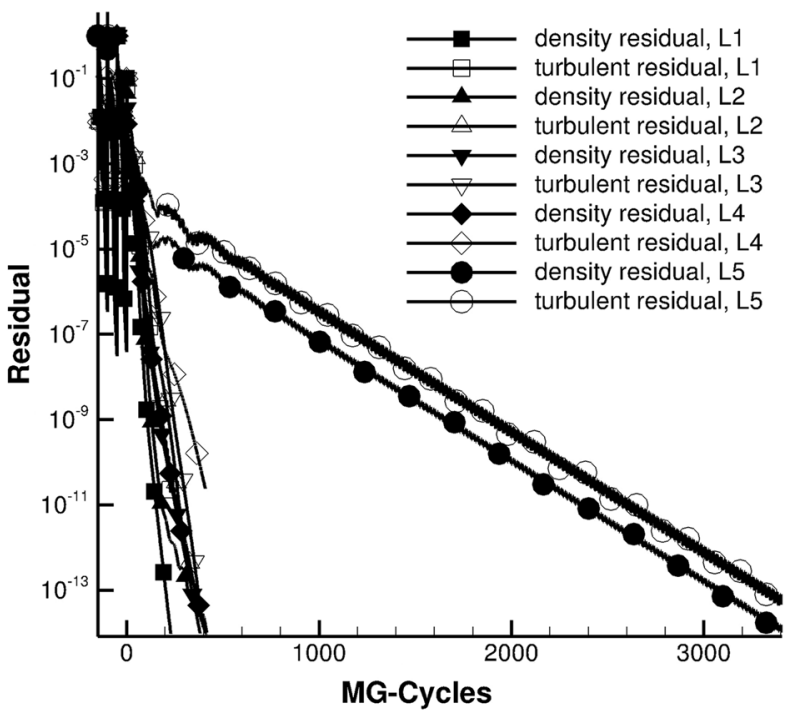

(b)

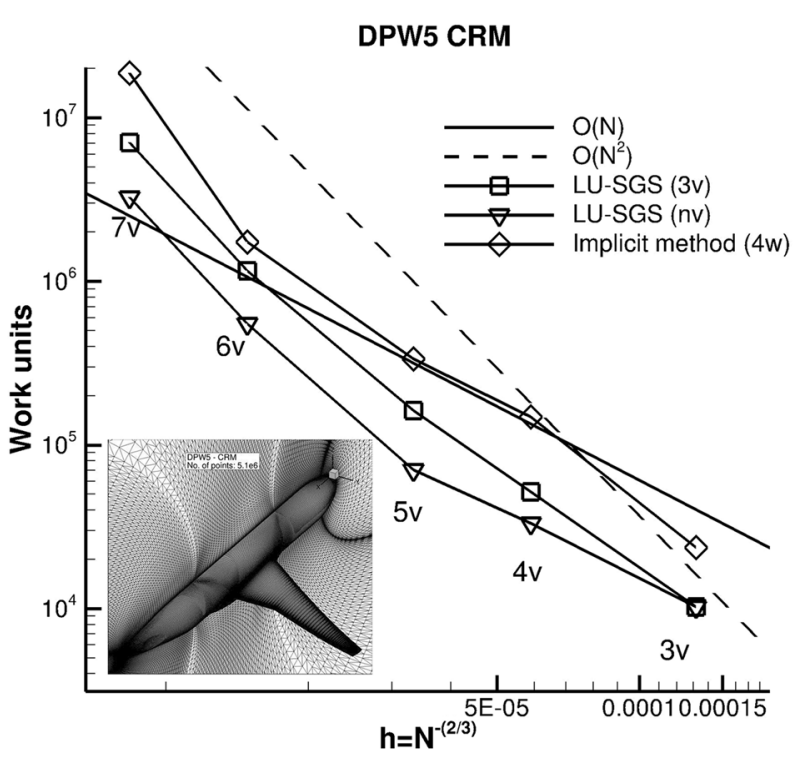

(d)

DPW5 CRM, LU-SGS

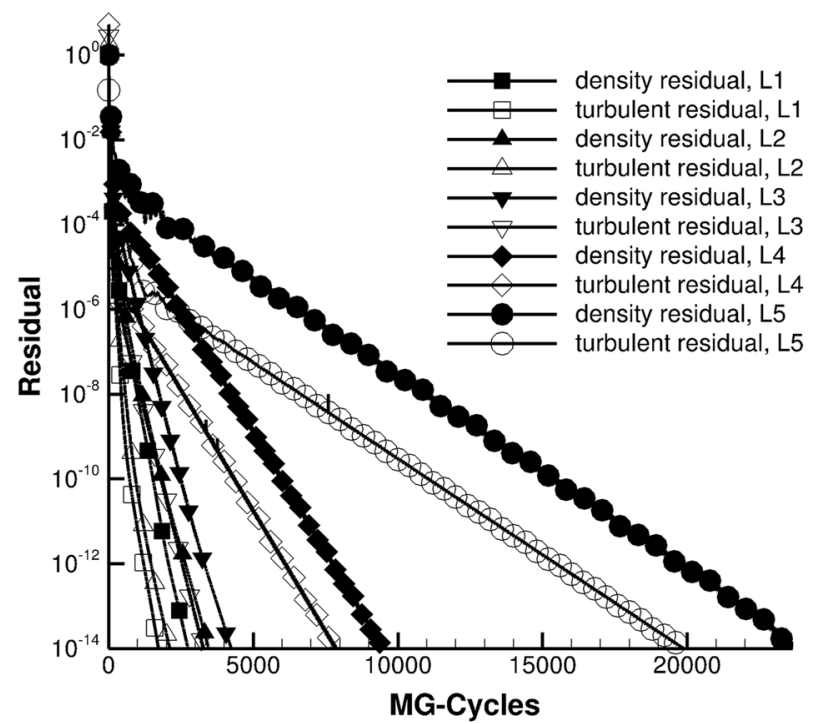

Fig. 3 Convergence behavior of implicit methods for the CRM wing/body-configuration $\left(M=0.85, \operatorname{Re}=5 \times 10^{6}\right)$ : a mesh, b work units as function of mesh size, $\mathbf{c}$ convergence history for implicit methods, $\mathbf{d}$ convergence history for LU-SGS method

An additional advantage is that certain features have only a small or no influence on the execution speed through the selective use of templates. Modern software development tools are used for the development, such as distributed version control (git), web-based code reviews (Gerrit) and continuous integration (Jenkins).

In the prototype to date a second order finite volume discretization and a higher order discontinuous Galerkin discretization were implemented on unstructured grids for the Euler equations with simple solution algorithms. The aim was to achieve the largest possible synergy between the two approaches. Next, the Flucs prototype will be extended to include additional functionality, for example RANS equations with one-equation turbulence model, coupling together several discretizations, SIMD and shared memory parallelization and improved implicit methods. These are not necessarily features that are the most important for users of flow solvers. On the other hand, they are aspects that potentially have a strong influence on existing software design. 
Fig. 4 Modular structure of the next generation flow solver Flucs
Time for an evaluation phase is planned after completion of the prototype, which will assess the decisions made, the design and its implementation. A first release for selected target applications in internal and external aerodynamic flows should be available at the end of the project.

\subsubsection{Parallelization}

Modern HPC systems have various degrees of parallelization at different levels: a network-connected cluster consisting of multiple computing nodes, each of which comprises several CPUs, each of which again has available multiple processing cores. A flow solver must optimize the use of all possible parallelization levels for a high-performance simulation. Domain decomposition provides the basis for parallelization, by which the simulation is carried out on multiple computing nodes. Computation on these domains is synchronized through exchange of data at the domain boundaries over the network. For best possible performance it is important to have a good balance both in computational load and in the necessary communication between domains. In the DLR TAU code significant improvements have been achieved in domain partitioning through the integration of graph-based algorithms, compared with partitioning based on recursive bisection. The TAU code uses domain decomposition as the sole parallelization strategy, with one domain per computing core. Figure 5 shows that this one-level parallelization is not optimal even for current HPC systems.

For a very small mesh $\left(2 \times 10^{6}\right.$ discretization points $)$ this one-level parallelization scales up to 480 processing cores before scaling breaks down at about 4000 discretization points per core due to increasing communication overhead as well as load imbalances (see Fig. 5, blue curve). A significantly improved scalability (red

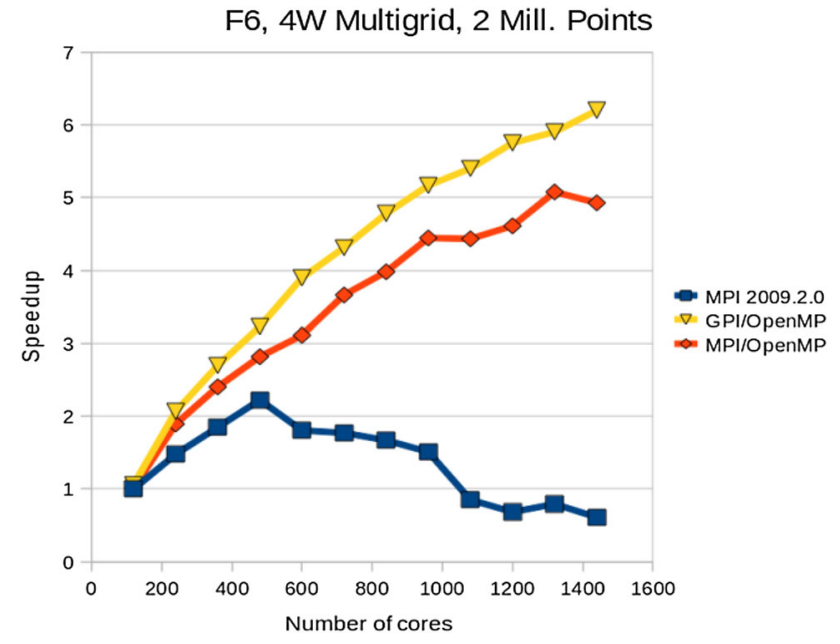

Fig. 5 Comparison of different parallelization strategies

curve) is possible as a TAU code prototype [17] demonstrates which uses one domain per multicore CPU. Here a two-level parallelization is used, where the domains are further subdivided so that a domain is now processed in parallel by all cores of a CPU, without (explicit) communication. This considerably reduces the comparatively slow communication. The scaling can be further improved by overlapping communication with computation (yellow curve). These findings form the basis for design of multi-level parallelization in the next generation solver Flucs. In addition to two-level domain decomposition there is a 3rd level of parallelization, namely the use of single instruction multiple data (SIMD) for unstructured meshes, which is a particular challenge addressed in Digital- $X$ to use current and future HPC systems efficiently for CFD simulations. 


\subsection{Reduced order aerodynamic models for loads analyses}

The loads analysis of an aircraft requires computation of thousands of parameter combinations. This includes variation of parameters describing the steady flow conditions in terms of Mach number, altitude and load factor, as well as parameters describing the flight dynamics, such as roll and pitch rate, and parameters defining the shape of the gust. Further, highly accurate predictions of the CFD code are required, in particular for transonic flow conditions. Their direct application for the entire flight envelope is, however, not practical due to the high computation times per calculation. Thus, reduced order models (ROM) are developed within the DLR Digital-X project to efficiently provide CFD-based aerodynamic data for loads analyses.

\subsubsection{Parametric ROM based on the Isomap method}

A parametric ROM for steady CFD data was developed based on isometric mapping (Isomap) [18], a nonlinear "manifold learning" (ML) method. It is assumed that the space of all CFD solutions forms a nonlinear manifold, which in turn constitutes a sub-manifold of $\mathbb{R}^{n}$ of lower intrinsic dimensionality. This is a more elaborate approach compared to the "proper orthogonal decomposition" (POD) method [19], in which a linear subspace of $\mathbb{R}^{n}$ is assumed. ML methods apply various approaches to identify the manifold geometry and to represent it in a low-dimensional Euclidean space. For this purpose Isomap uses the pairwise geodesic distances between previously generated CFD solutions at selected parameter combinations. After approximating these distances Isomap computes a data set of low-dimensional vectors in the so-called embedding space, whose pairwise Euclidean distances correspond to the approximated geodesic distances. A mapping from the embedding space back onto the manifold in the high-dimensional CFD solution space was developed to make predictions of CFD solutions at any point in the parameter space. When combined with an interpolation model between the parameter space and the embedding space, similar to POD with interpolation (POD + I, [20]), an Isomap-based ROM, called Isomap + I [21], is obtained.

Figure 6 provides a comparison of the predictions of Isomap + I and POD + I with the computed TAU reference solution for the pressure coefficient at three wing sections for the LANN wing [22] in inviscid flow. Isomap + I yields better predictions than a POD-based interpolation method, in particular for the location and magnitude of the shock wave at transonic flow conditions. The parameter space, defined by variations of the angle of attack, $\alpha$ and the Mach number, M, was previously sampled in the range $[\alpha \times \mathrm{M}]=\left[1^{\circ}, 5^{\circ}\right] \times[0.76,0.82]$ using a
"Design of Experiment" (DoE) based on a Latin/Hypercube approach with 25 different $\alpha$-Mach combinations. At these parameter combinations the corresponding CFD solutions were computed and used as input data for both ROMs. The required parameters for Isomap $+\mathrm{I}$ were determined automatically.

\subsubsection{Correction to the doublet lattice method}

CFD solutions for selected parameter combinations have been used to correct the doubletlattice method (DLM), the standard method for calculating aerodynamic loads in aeroelasticity. The DLM solves the linear potential equations under the assumption of isentropic, inviscid flow, neglecting thickness effects. Thus, the method is valid only for subsonic flow conditions. Shocks and flow separation cannot be represented by the method and a correction is required in the transonic velocity range.

The DLM correction method CorrREcting Aerodynamic Matrices (CREAM) [23] was developed to improve predictions for unsteady aerodynamic loads. In addition to a quasi-steady correction (CREAM-0) an unsteady support point can be considered (CREAM-1). The more accurate solution (RANS method) and the faster approximate solution (DLM) are expanded as Taylor series with respect to the reduced frequency. The Taylor coefficients of the lowfidelity series are successively replaced by coefficients of the more accurate series. By further transposition the explicit Taylor series can be circumvented. In addition, it is assumed that the solution of the more exact RANS method can be represented by the AIC matrix (Aerodynamic Influence Coefficient) through multiplication by a correction matrix.

The additional correction term in CREAM-1 improves the phase component of the frequency response, thus enlarging the region of validity of the correction along the frequency axis. This is demonstrated in Fig. 7, using the example of a forced pitching motion. The calculated frequency response with DLM significantly underestimates the magnitude, with the phase response also shown incorrectly. CREAM-0 significantly improves the predictions of the magnitude. The correction also improves the phase, although it is not sufficient. The phase curve is corrected in CREAM-1 due to the additional frequency reference point, although the effect on the magnitude is fairly small. With increasing frequency, the deviation increases between the CREAM solutions and the nonlinear reference CFD solution. This can be explained because the CFD support points used in the corrections are at zero frequency, or close to zero, thus extrapolation to higher frequencies is represented by the DLM model only.

The method CREAM-0 was also used to calculate the aerodynamic response of " $1-\cos$ " gust loads. As a 

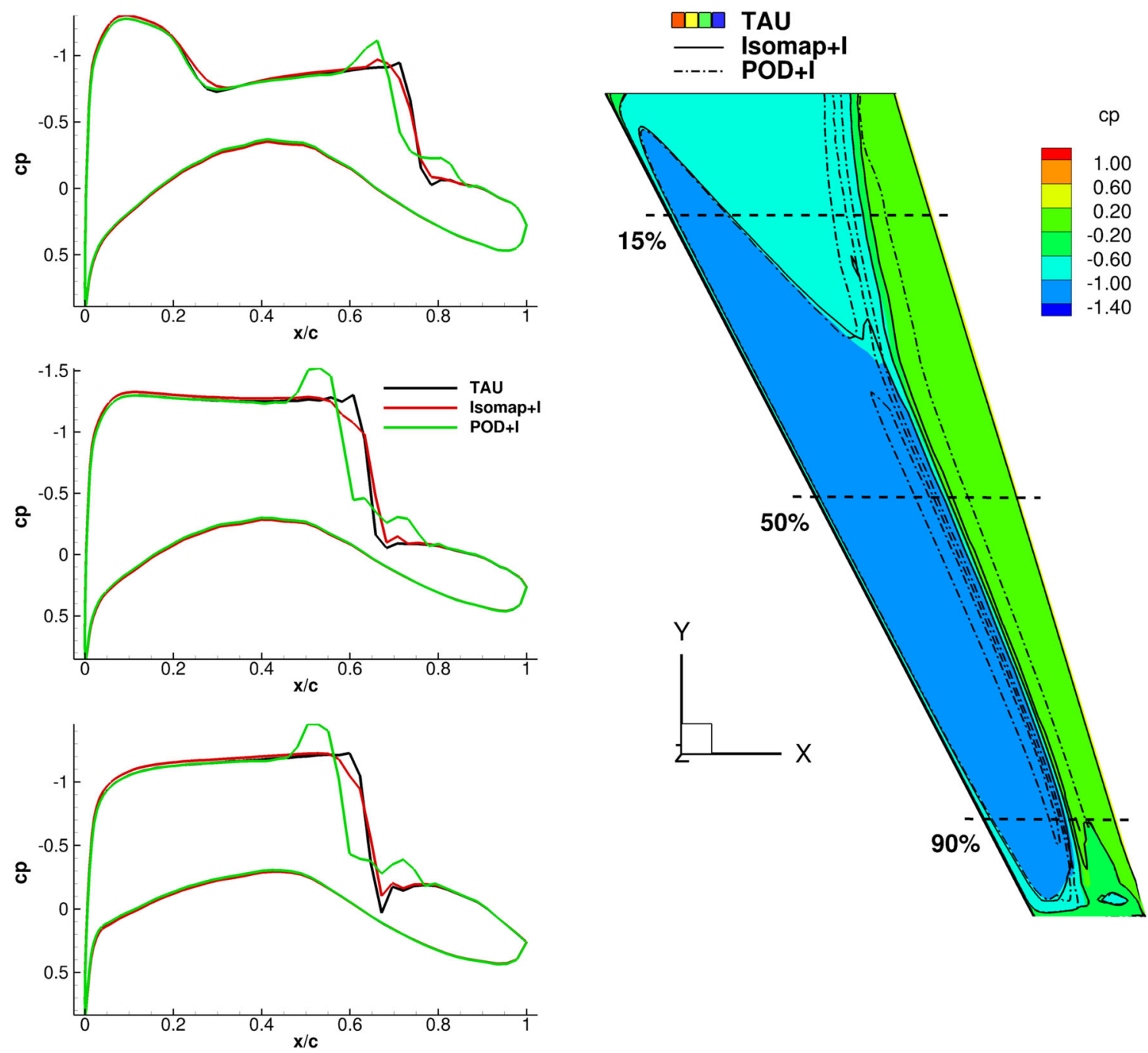

Fig. 6 Steady Euler computation for the LANN wing compared to ROM predictions

reference a gust load was simulated using the Field Velocity Method (FVM) [24] with the nonlinear CFD solver TAU. The gust load was calculated with both DLM and CREAM-0. The correction data were computed with the linearized TAU code in the frequency domain (LFD code, [25]) applying a rigid pitching motion at zero frequency. The effect of the correction can be clearly seen in Fig. 8. Compared to the nonlinear CFD solution, the DLM predicted amplitude is too low at these transonic flow conditions. In contrast, the result obtained by CREAM-0 agrees very well with the reference solution.

\subsection{Software platform for multidisciplinary optimization of the complete aircraft}

The main objective of this activity is the development, implementation and testing of suitable strategies for multidisciplinary optimization (MDO) of the complete aircraft based on highly accurate numerical methods. One major activity is therefore extending the MDO capabilities that were initially developed for preliminary design in DLR internal projects TIVA I/II [27] and VAMP [28] to highfidelity methods. Initial work took place in the DLR project MDOrmec [29]. In Digital-X, a flexible software platform based on the parallel simulation environment FlowSimulator [30] (see Sect. 3.7.1) is being developed by considering tools and processes from all relevant disciplines. This platform will provide a robust, integrated design process for aerodynamics and structures, while taking the engine into account. To use the full potential of multidisciplinary design the idea is to replace the predominantly sequential approach to detailed design by pursuing a "multi-level" approach, which combines highly accurate multidisciplinary analysis (MDA) and MDO processes for 
aerodynamics and structures with simplified techniques for complete aircraft design and rapid methods for the identification of critical load cases. Rule-based design procedures are implemented for preliminary design. Coupling of the individual tools and components and execution of the process chain is controlled by DLR's workflow management tool RCE (Remote Component Environment) [31]. The description of aircraft geometry and exchange of data between components are achieved using DLR's XMLbased Common Parametric Aircraft Configuration Scheme (CPACS) [32]. The aircraft geometry is modeled using DLR's geometry library TIGL and various toolspecific model generators which are able to read and write the CPACS data format. In the frame of Digital-X project focus is put on the optimization of conventional aircraft configurations.

\subsubsection{MDO architecture and process chain}

Figure 9 shows schematically the sequential MDO process chain that has been developed. The formulation is considered a per-cycle "multidisciplinary feasible" approach, coupling the involved disciplines at different stages of fidelity. The process is driven by a single, gradient-free optimization algorithm, where all objective-relevant data are provided by the detailed level (i.e., coupled high-fidelity methods).

The chosen "multi-level/variable fidelity" MDO approach allows for an efficient treatment of configurational design requirements by means of preliminary design methods. Assessment of configuration-specific critical load
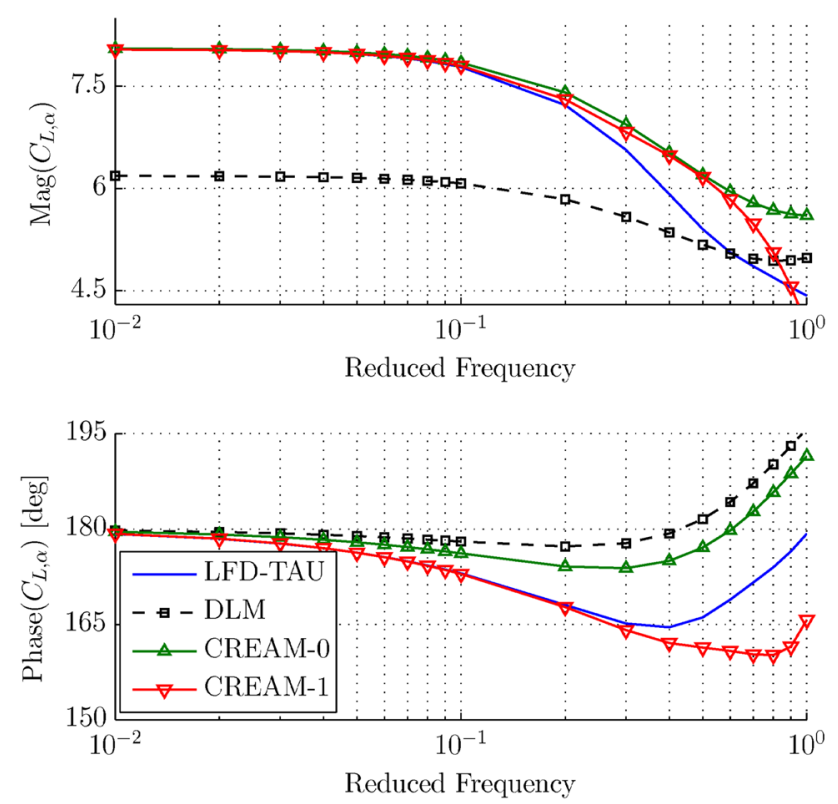

Fig. 7 Frequency response $\mathrm{dCL} / \mathrm{d} \alpha$ for a pitching motion on the LANN wing, $\mathrm{M}=0.82, \alpha=0.6^{\circ}$ cases and initial structural sizing is done in the second stage. Employing high-fidelity coupled CFD/CSM (computational structural mechanics) simulations and refined structural sizing, the detailed level provides the required performance data for subsequent objective function evaluation. This automated process chain is currently being implemented as a prototype in DLR's distributed, simulation integration environment RCE.

\subsubsection{Reference configuration}

The Airbus XRF-1 configuration is used as the reference geometry to demonstrate the capabilities of the MDO environment in a realistic application. The XRF-1 is a research configuration similar to an existing Airbus widebody aircraft. Figure 10 shows the XRF1 geometry as a wing/fuselage/tail configuration. It was specified consistently in CPACS format and recalculated using improved preliminary design tools.

Figure 11 shows results for the recalculation of the reference mission. This is a simplified $8000 \mathrm{~nm}$ mission consisting of climb, cruise, descent and landing as well as a flight to an alternate airport $(200 \mathrm{~nm})$. The results obtained with DLR's preliminary design tools perfectly match the reference results by Airbus, demonstrating that a consistent definition of the geometry and top-level aircraft requirements was specified.

\subsubsection{Loads and structural sizing}

For efficient calculation of critical structural design loads and load cases the Dynamic Master Model (DMM) of the XRF-1 configuration was generated [33]. Figure 12 shows the applied finite element (FE) model and the aerodynamic model which were created automatically from data in the corresponding CPACS file. The detailed Static Structural

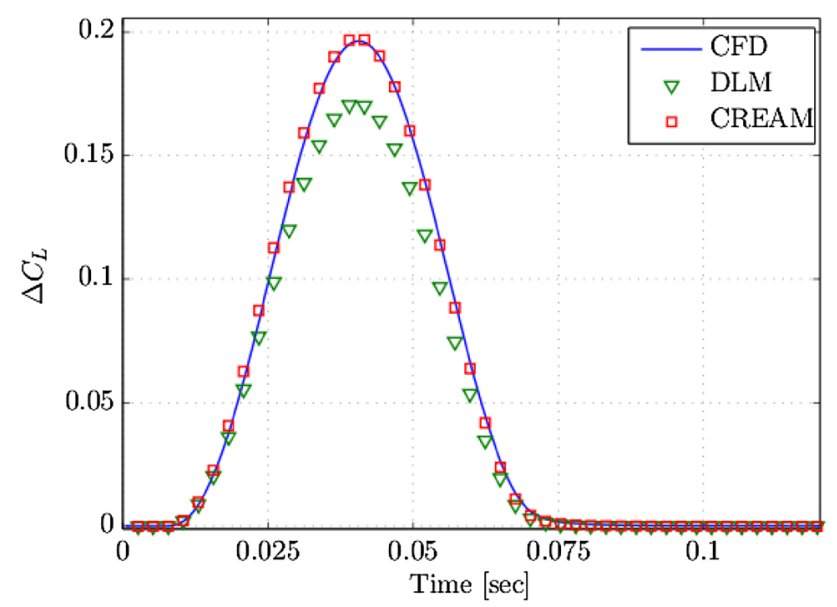

Fig. 8 Aerodynamic response of the lift coefficient for a "1-cos" gust for the Aerostabil wing [26], $M=0.8, \alpha=0.0^{\circ}$ 
Fig. 9 Digital-X MDO process chain (schematic)
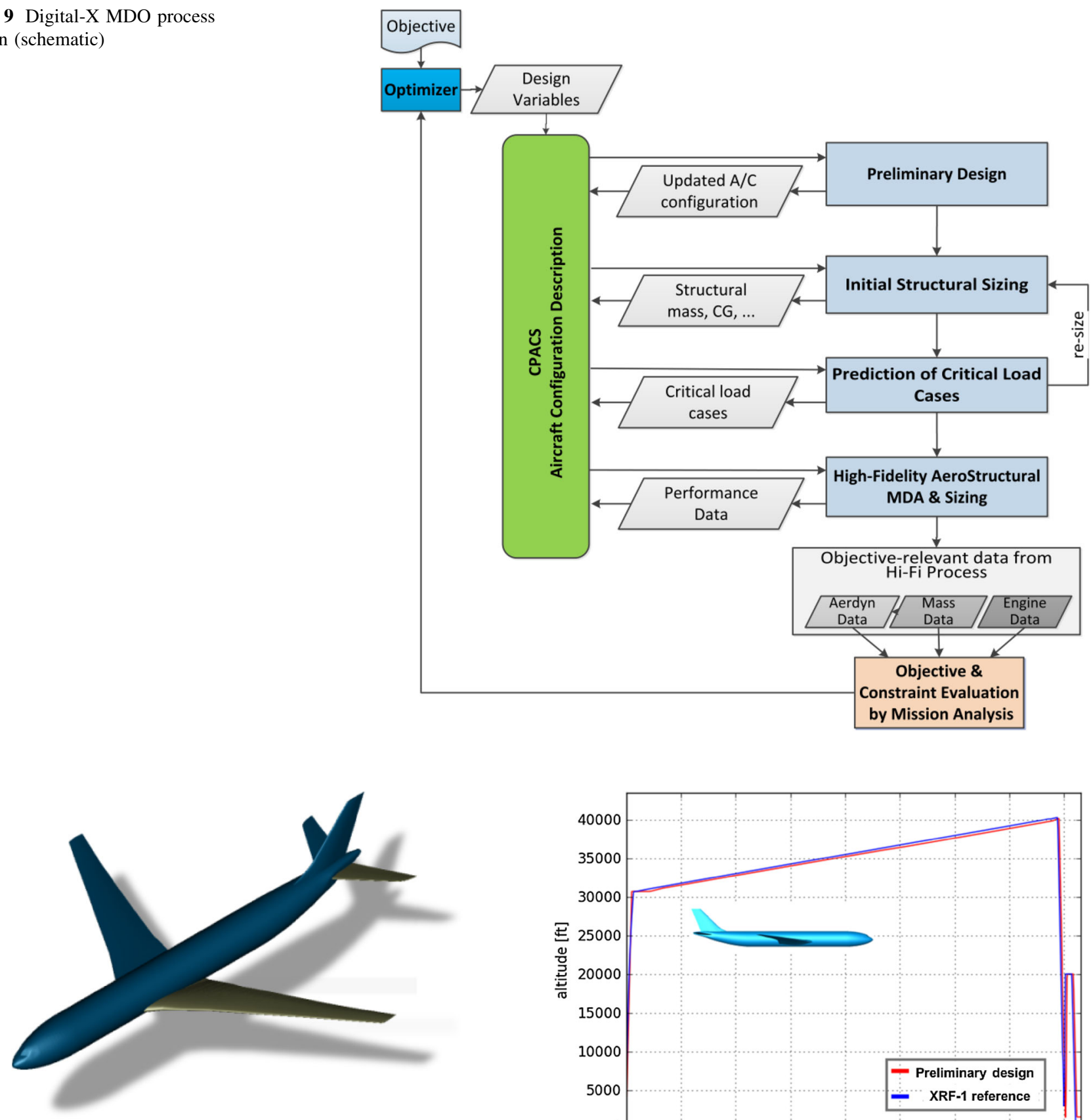

Fig. 10 XRF-1 reference configuration

Model (SMM) for the XRF-1 wing/fuselage configuration for structural analysis in metallic design was also created with advanced, automated model generators [34] from the CPACS file and is shown in Fig. 13. The model generators were developed in previous projects and have been enhanced within Digital-X to match the requirements of the MDO process. The structural sizing of the SMM is based on critical loads calculated with the DMM, as well as those recalculated for selected critical load cases using highly accurate methods. Work is also under way to extend the static and dynamic structural analysis tools and model generators to deal with

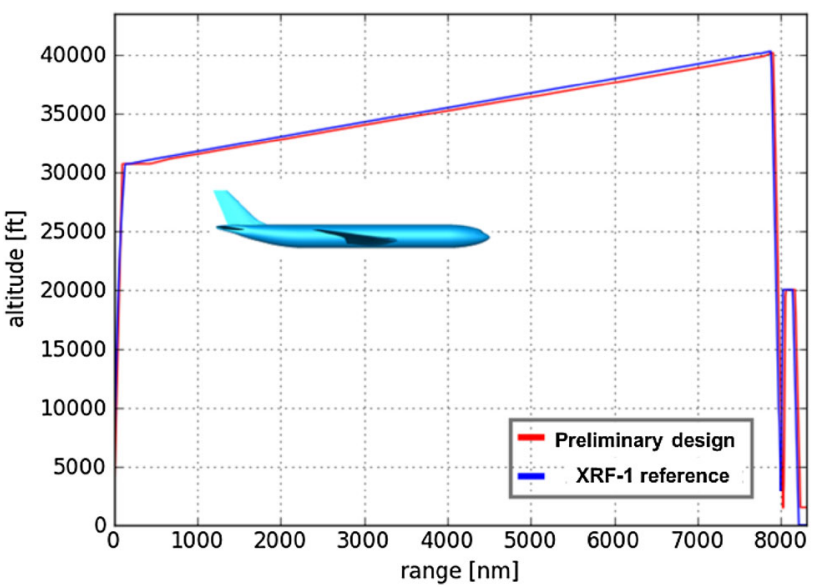

Fig. 11 Recalculation of long-range mission with preliminary design tools

composite structures. Results for sizing the composite XRF1 wing, fuselage and horizontal tail plane based on 17 generic DMM load cases are available.

\subsubsection{Validation and demonstration}

After specifying the optimization tasks, the capabilities of the MDO process were first demonstrated using a 
(a)

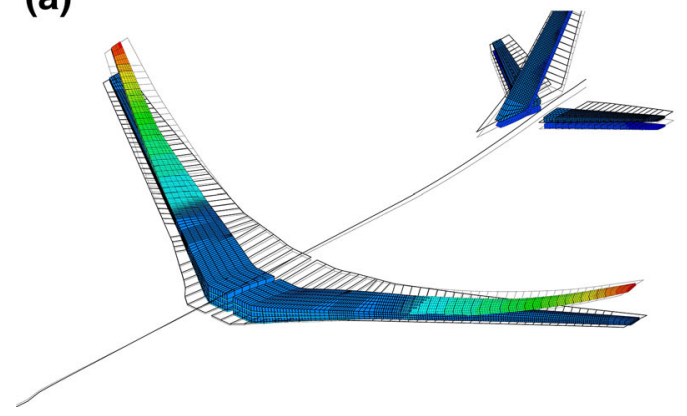

(b)

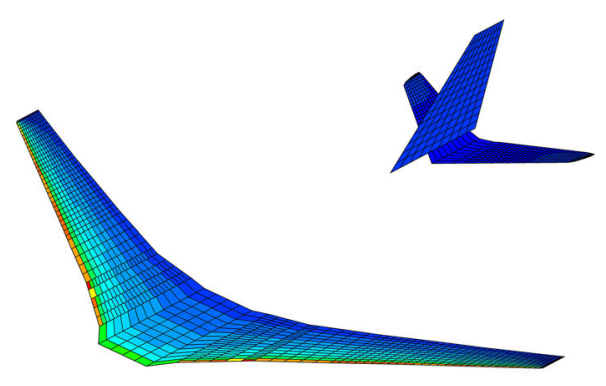

Fig. 12 Dynamic model of XRF-1: a global FE model for modal analysis (first wing bending), b doublet-lattice model with pressure distribution

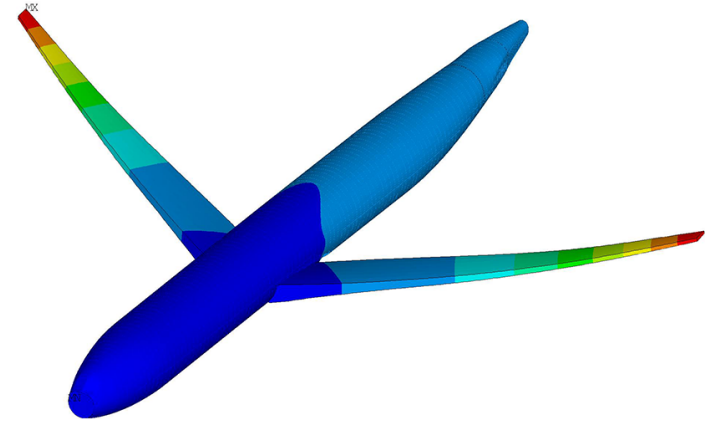

Fig. 13 Detailed coupled structural model of XRF-1 configuration in metallic design

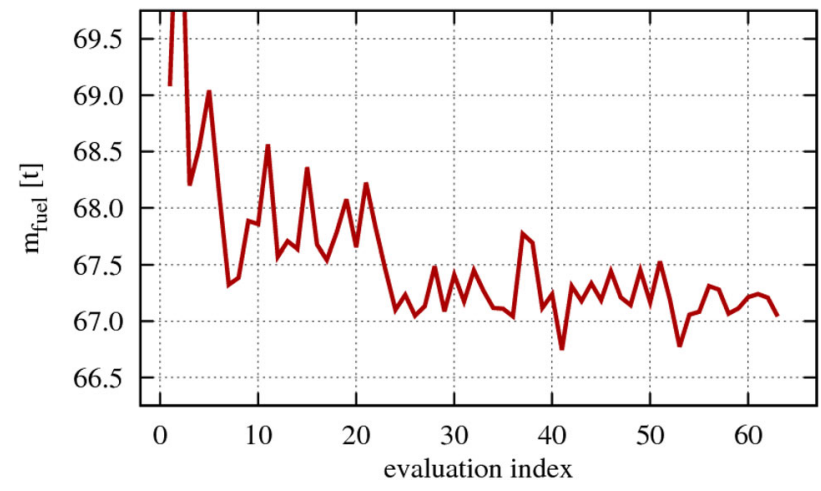

Fig. 14 Optimization convergence history relative to baseline block fuel mass

simplified XRF-1, consisting of wing and fuselage. Figures 14 and 15 show results of a multidisciplinary optimization of the block fuel mass.

The fuel consumption was reduced by $3.6 \%$. This result was achieved with the MDO chain on the detailed level, using a Subplex algorithm. High-fidelity CFD calculations were used for the wing/fuselage aerodynamics, coupled with a finite element analysis of the wing structure to

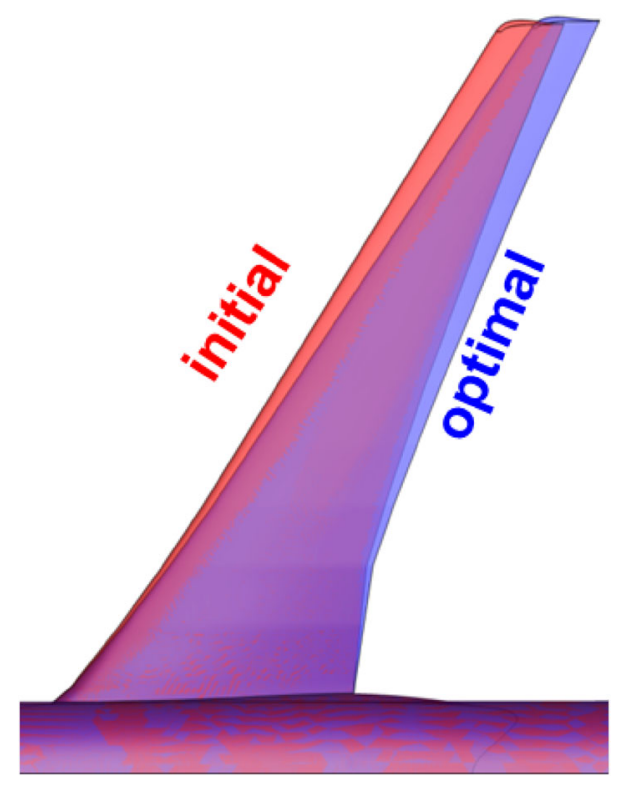

Fig. 15 Result of multidisciplinary optimization of XRF-1 wing/fuselage configuration

determine the static aeroelastic equilibrium. In addition, the wing structure was sized using two predefined load cases for each optimization step. The wing was parameterized using five geometry parameters (twist, aspect ratio and sweep) with planform area kept constant. Many other constraints that are necessary to achieve a realistic aircraft design were not yet taken into account at this time. For the evaluation of the objective function a mission analysis based on backwards-integration of an ordinary-differential equation (ODE) for a simplified mission over $5600 \mathrm{~nm}$ with a three-segment cruise climb from 11000 to $12000 \mathrm{~m}$ was carried out.

\subsubsection{Gradient-based optimization}

To improve efficiency, gradient-based optimization methods are used which make possible a large number of design 


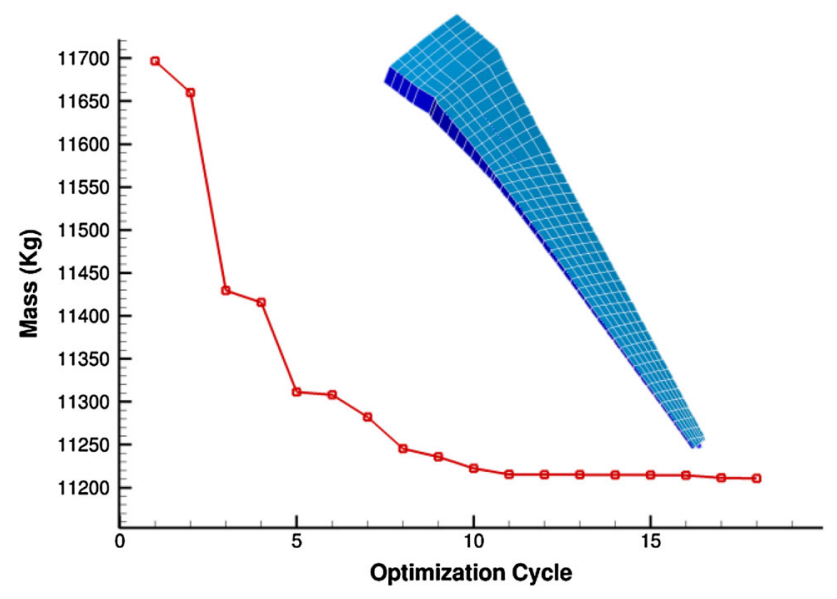

Fig. 16 Gradient based optimization of the wing structural mass

parameters. Figure 16 shows the first results of a gradientbased structural optimization of a wing with 245 regions optimized and 5880 constraints. In this case the structural mass was optimized considering four different failure criteria and 12 load cases.

For this purpose, the suitability of various optimization algorithms was tested with a single objective function and many constraints. A sequential quadratic programming (SQP) method was applied here with gradients calculated using finite differences. As a next step the entire MDO process chain is to be converted to a gradient-based optimization.

In future developments separation methods are being followed in addition to gradient-based methods, which decouple sub-processes so that multiple optimizers can be used. The full possibilities of multidisciplinary optimization based on the "multi-level/variable fidelity" approach will be demonstrated with additional parameters and constraints, a more complex mission, and the trimmed complete XRF-1 aircraft configuration. Further design load cases are also to be considered in the context of the automated sizing of the complete aircraft with carbon fiber reinforced polymer composites (CFRP). Correction techniques and reduced order models should be applied to improve efficiency and accuracy in the loads process. Finally, the MDO processes developed are being evaluated and the optimization results are analyzed with regard to their physical improvements. At the end of the project a best practice guideline for multidisciplinary optimization of the complete aircraft will be delivered.

\subsection{Simulation of flight maneuvers on the borders of the flight envelope}

The analysis of maneuver loads is intrinsic to the design and certification of aircraft. The aim of this activity is to provide methods and processes for the numerical simulation of free-flying elastic aircraft. For the demonstration of the capabilities of the methods developed, extensive and challenging simulation scenarios are planned, particularly on the borders of the flight envelope. Unlike the methods that are primarily in use in industry at present for flight dynamics analysis and load calculations, which in most of the cases are based on fast, yet very simplified aerodynamic methods, Digital-X puts emphasis on the development and coupling of high-fidelity simulation methods using CFD and CSM codes.

\subsubsection{CFD/CSM coupling}

The flight dynamics simulations intended in Digital- $X$ involve computations of the aircraft's aerodynamics using $\mathrm{CFD}$, its structural dynamics using computational structural
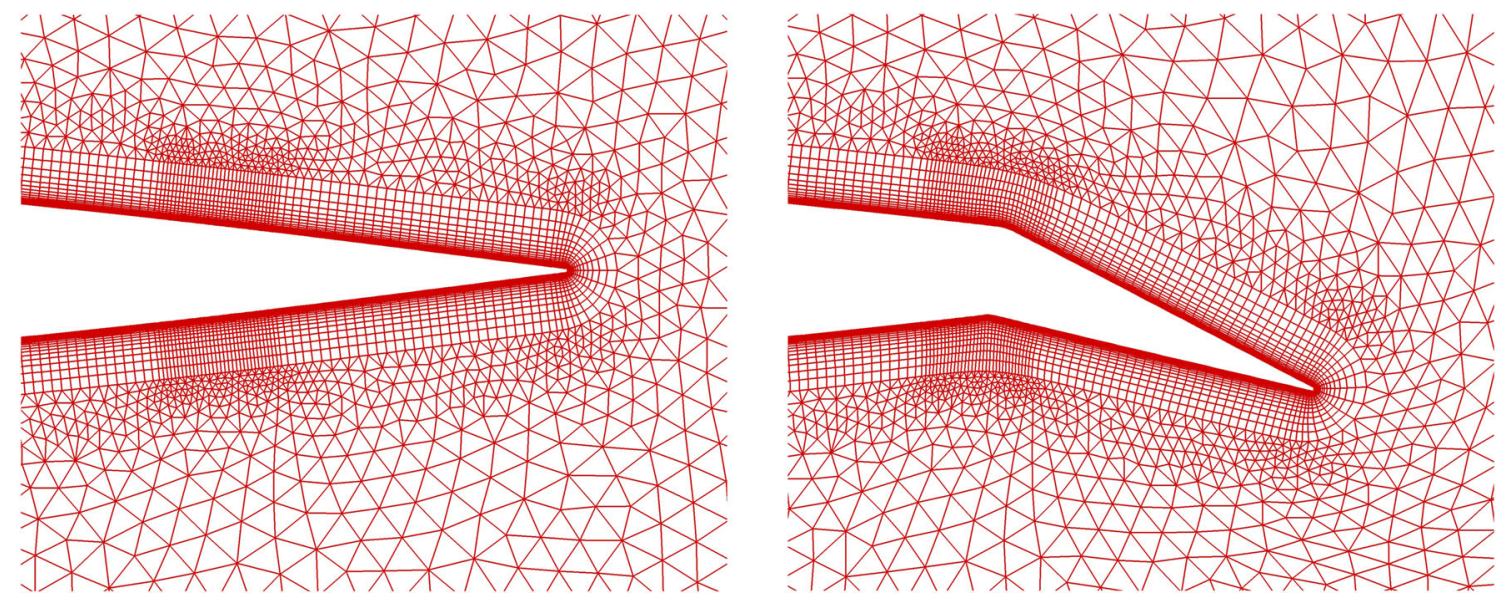

Fig. 17 Deformation of the volume mesh around an airfoil-rudder configuration (without gap modeling) using the RBF technique 
mechanics (CSM), and its flight mechanics (FM) and flight control. The computations are regarded as a multi-fields problem, that is, independent and highly specialized solvers for the single-field problems are suitably coupled together in space and time under consideration of the boundary conditions mutually existing between the single-field problems. The term spatial coupling refers to the operation that performs the required energy-conserving transfer of aerodynamic loads from the CFD surface mesh to the structural model, and, vice versa, the reverse transfer of structural deformations. The temporal coupling represents the appropriate synchronization of the individual singlefield solver calls. In Digital-X, both tight and loose coupling schemes are foreseen. The former ensures correct energy transfer by performing several sub-iterations between the single-field solvers at each time step while the latter gives only an approximately correct energy transfer, but is less computationally intensive. In an ensuing step to the transfer of coupling data, the entire CFD volume mesh needs to be adapted to the deformation state of the CFD surface mesh. High requirements are set for this operation in terms of computational cost and preservation of mesh quality. Both requirements are difficult to fulfill, particularly in applications to complex geometries which involve control surface deflections and are subject to large deformations. Nevertheless, substantial progress has been made within Digital$\mathrm{X}$ on further development and improvement of the mesh deformation using Radial Basis Functions (RBF).

\subsubsection{Modeling control surfaces}

In CFD-based simulations of aircraft maneuver, the capability is needed to carry out (time-dependent) motions of the primary control surfaces. Similarly in steady simulations of the aircraft trim state, (static) control surface deflections must be considered, at least of the horizontal tail plane. In recent years, considerable progress has been made on modeling movable control surfaces in previous projects [35] and in Digital-X. An established and robust method for the rotation of control surfaces is to use mesh deformation, which avoids the challenging, complex and computationally intensive application of the overset grid technique (also known as the Chimera method). The vortices emanating from gaps between the control surfaces and the main lifting surface, along with the circulation changes induced by them, are not considered in the procedure that is solely based on mesh deformation, since the gaps cannot be modeled. However, the main effect of the deflection may be captured very efficiently in this way and usually with sufficient accuracy. As an example, the deflection of a generic rudder using RBF-based mesh deformation is shown in Fig. 17. The quality of the original non deformed CFD volume mesh is maintained
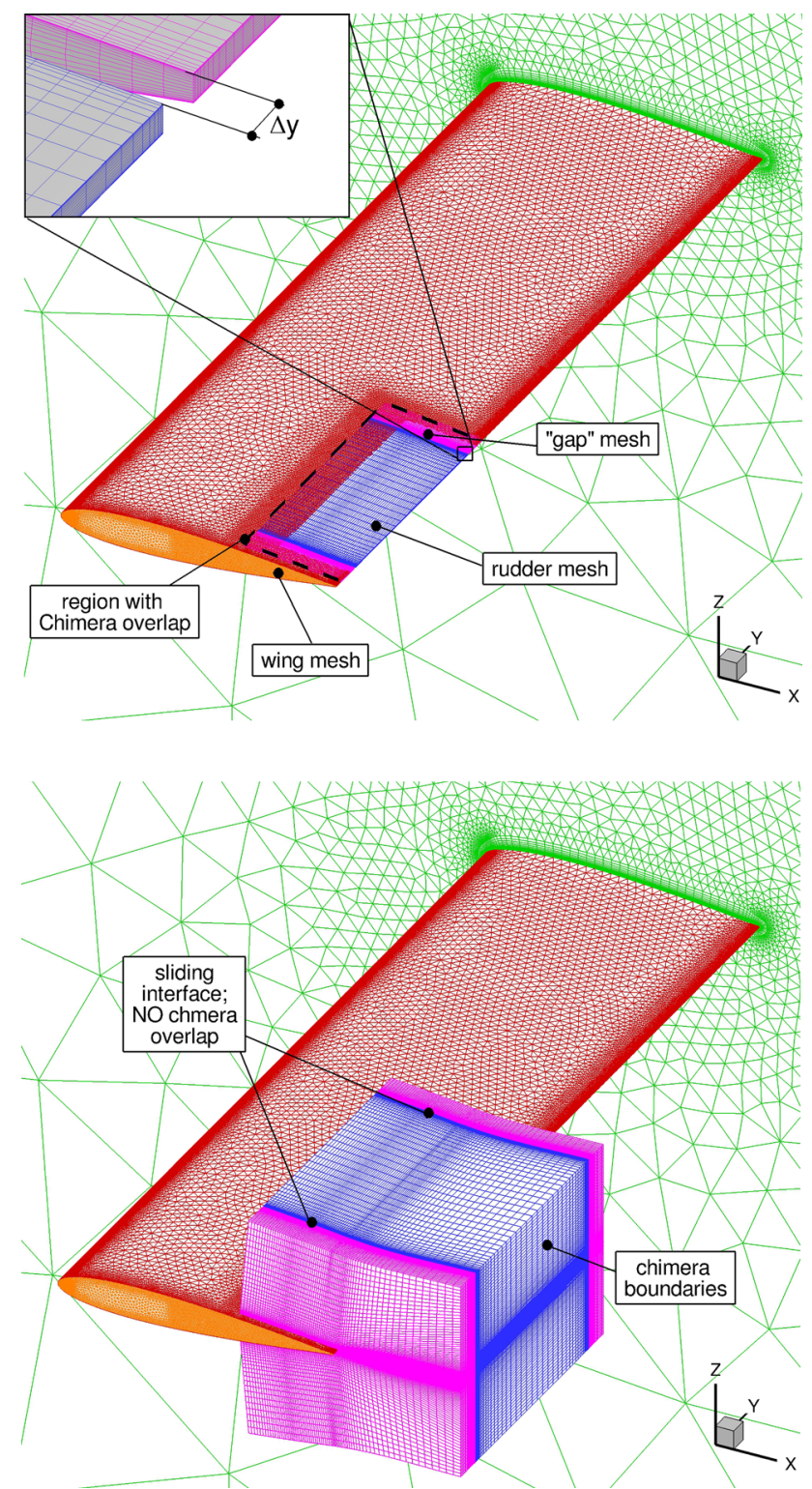

Fig. 18 Application of the "sliding interface" technique for installation and deflection of a rudder

during mesh deformation, even at very high rudder deflections.

A further enhancement for modeling movable control surfaces in Digital-X is the combination of the so-called "patched grids" method with the RBF-based mesh deformation. In contrast to pure mesh deformation techniques, this method can conveniently take into account existing rudder gaps. The basic procedure of the technique is illustrated in Fig. 18 for a generic aileron. The control surface installation is based on four meshes. The wing mesh serves as a background mesh. The actual rudder and half of the gap to the wing on each side are embedded in an individual Chimera mesh. Two extra Chimera meshes 
represent the other halves of the rudder gaps, including the adjacent parts of the wing mesh. The meshes do only overlap along the positions indicated in Fig. 18. Without the patched grid method, additional Chimera meshes would be needed between the rudder and the neighboring gap meshes, see the planes marked by "sliding interface" in Fig. 18. In the initial state without rudder deflection, the mesh points on both sides of the sliding interface planes are matching. The new algorithm automatically generates sufficient mesh overlap in the gap region by mesh
Fig. 19 a Deflected generic aileron modeled by "sliding interface" technique, b resulting CFD solution ( $c_{\mathrm{p}}$ and eddy viscosity)
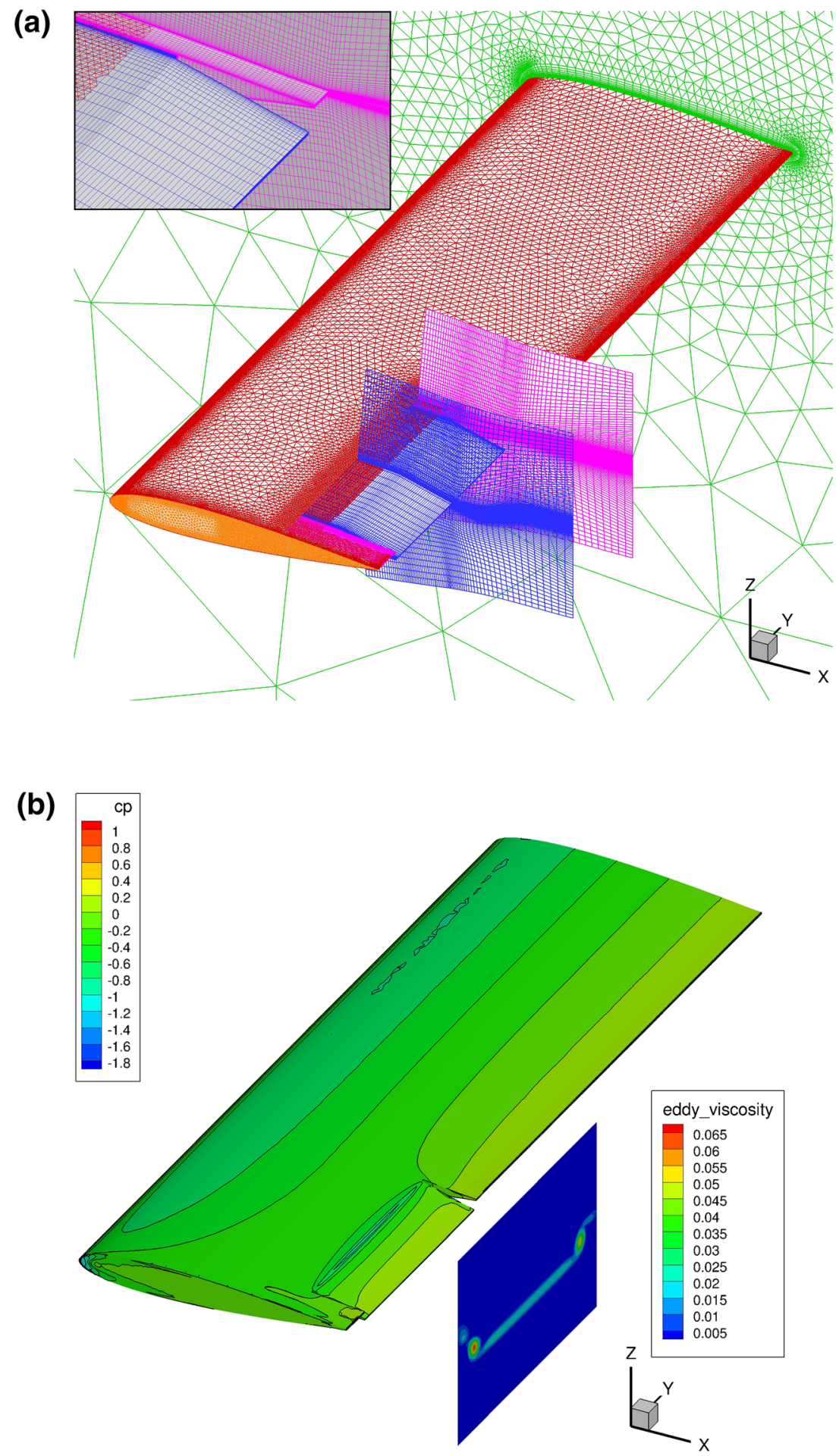


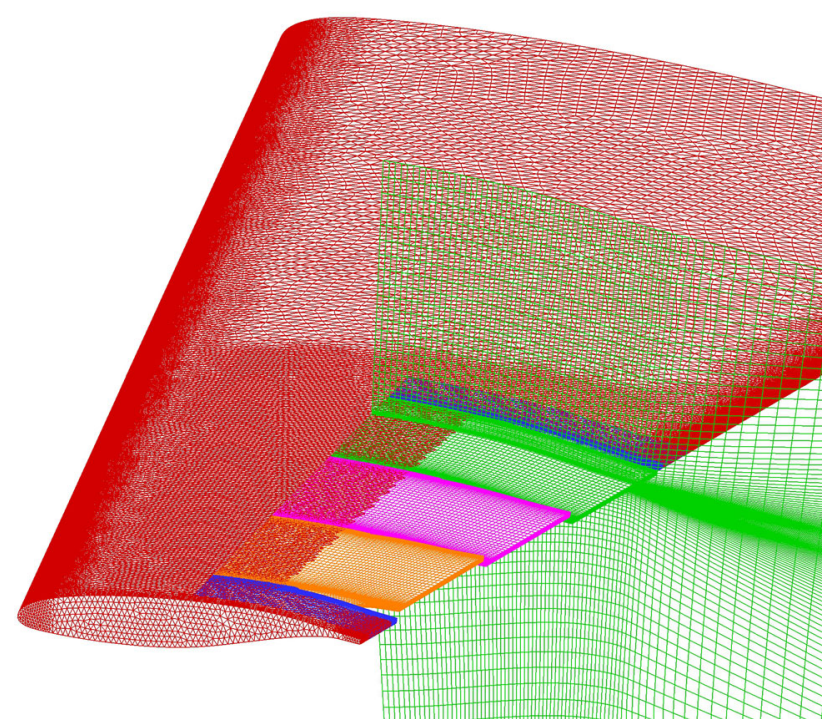

Fig. 20 Three-part aileron modeled by "sliding interface" technique

extrusion. In situations with flap deflection, interpolation of the flow quantities in the gap region is performed using the Chimera technique.

For actually deflecting the flap, the RBF-based mesh deformation is applied to the mesh containing the flap only. In this operation, the outer boundaries of the Chimera meshes always remain fixed (see Fig. 19) so that the CPUintensive Chimera search for donor cells only needs to take place once. The patched grids technique avoids the cumbersome and often very time-consuming manual generation of meshes having sufficient overlap in the rudder gap regions such that the Chimera mesh technique is applicable. Furthermore, many mesh points are saved by the technique, which would otherwise be required solely to ensure the Chimera overlap in the gap regions. This considerably reduces computing time compared with a pure non-automated Chimera-based control surface mesh setup. The new method is extremely flexible in its application and for example can also be used for modeling a segmented aileron (Fig. 20).

\subsubsection{Flight dynamics module}

A flight dynamics module has been developed for the unsteady simulation of an elastic aircraft in the time domain. This module provides the necessary spatial and temporal coupling of aerodynamics, structural dynamics, and flight mechanics. It is implemented in Python and uses the FlowSimulator framework for massively parallel computations. The translational and rotational motion of the center of gravity of the aircraft is calculated by

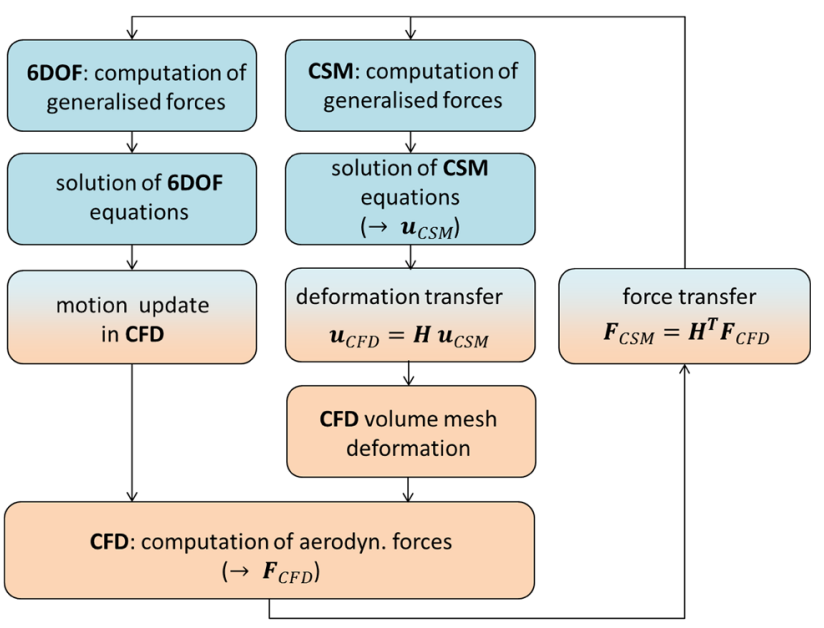

$\boldsymbol{H}$ : CFD-CSM interpolation matrix

Fig. 21 Schematic representation of the time integration loop in the flight dynamics module for spatial and temporal coupling of aerodynamics (CFD), structural dynamics (CSM) and flight mechanics $(6 \mathrm{DOF})$ codes

Newton's second law and Euler's gyroscopic equations. For the decoupling of the rigid body and elastic degrees of freedom, the equations are formulated in a coordinate system which obeys the so-called "mean axes" conditions. The integrated structural dynamics solver operates under the assumption of a geometrically and physically linear structural behavior. The equations of motion of the free-flight elastic aircraft were derived from Hamilton's principle. They were spatially discretized with the finite element method and transformed into modal space using the elastic modes of the unconstrained aircraft. Time integration is carried out with the Newmark scheme. A "scattered data" interpolation with a thin plate spline RBF is provided by the flight dynamics module for the transformation of the aerodynamic loads onto the structural grid and for the interpolation of structural deformations onto the CFD grid. Figure 21 illustrates the loop implemented in the flight dynamics module for time integration of the multi-field problem described.

As an example Fig. 22 shows the application of the flight dynamics module for simulating the interaction of an elastic aircraft with a "1-cos" gust. The plot shows the heave and pitch accelerations as function of time. The interaction of the elastic deformation with the rigid body motions of the aircraft becomes evident as higher frequency vibrations.

The initial condition for the simulation of response problems such as gust interaction is typically an horizontal flight with constant velocity. This initial (trim-) condition is computed by the flight dynamics module with a Newton 
(a)

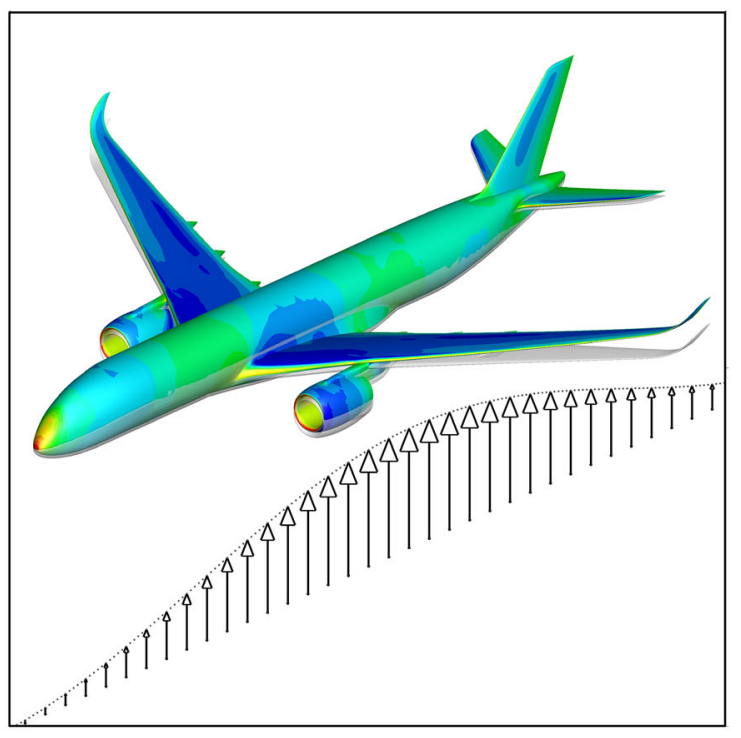

(b)
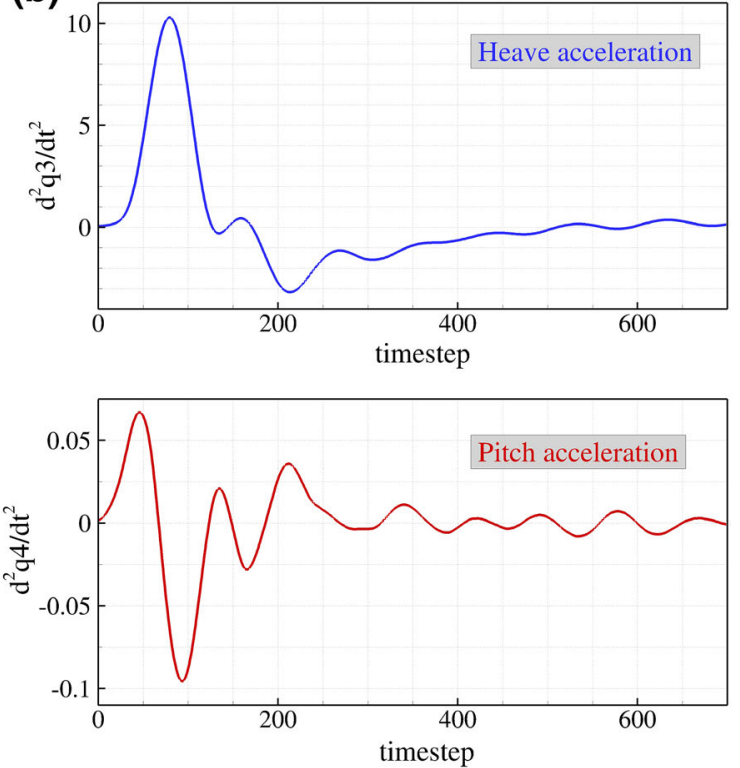

Fig. 22 Interaction of a transport aircraft with a gust: a instantaneous elastic deformation and initial state (gray) corresponding trim solution, b heave and pitch accelerations at the center of gravity

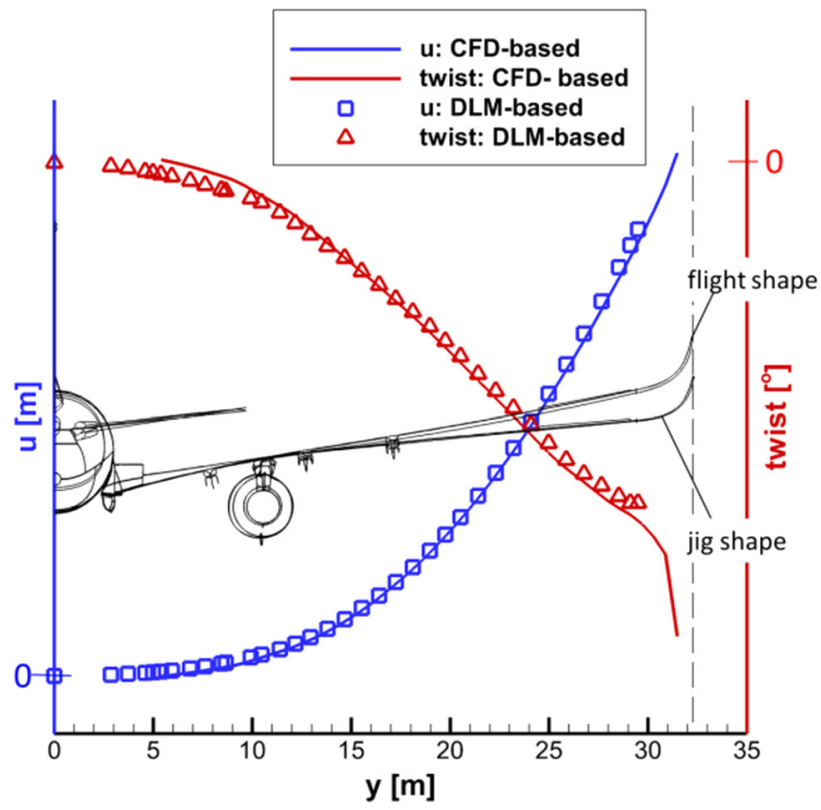

Fig. 23 Spanwise bending and twist distribution of the trimmed aeroelastic equilibrium configuration, as predicted by an industrial reference process and the Digital-X process (subsonic test case)

algorithm which iteratively solves for static aeroelastic equilibrium.

\subsubsection{Simulation scenarios}

In the context of Digital-X, aiming for more precise predictions of aircraft loads, a set of maneuver scenarios is addressed using highly accurate CFD-based simulation methods. During certification, proof must be provided that the designed aircraft structure withstands the loads occurring in these scenarios. As an example, the simulation of one scenario is discussed in detail below-the gust encounter of a modern elastic passenger aircraft.

To evaluate the potential of a CFD-based load analysis process, Airbus started the so-called CFD4Loads initiative in 2013. DLR was involved in this initiative, besides Universities and other European research organizations such as ARA and ONERA. Investigations were carried out on a realistic passenger aircraft test case (Fig. 23). The focus of DLR was on simulating gust interactions. Six gust load cases were considered. They differ in terms of Mach number, Reynolds number, flight altitude and aircraft weight, as well as gust wavelength, gust amplitude and direction (upwind or downwind). So far, only gusts which influence the aircraft longitudinal motion have been considered. In the following, exemplary results are presented for an investigated load case in transonic flow $(M=0.836$, $R e=86 \times 10^{6}$ ). An upwind gust of about $12 \mathrm{~m} / \mathrm{s}$ and a penetration depth of $350 \mathrm{ft}(107 \mathrm{~m})$ acts on the aircraft with low flight mass $m=150 \mathrm{t}$. Compared to the load cases with subsonic flow, which also were investigated in CFD4Loads, the transonic case is of particular interest for CFD-based load analysis, since conventional process chains that are based on linear aerodynamics are likely to reveal their limitations in the transonic flow region. An important aspect of the CFD4Loads initiative was to 
examine whether the predicted gust loads are affected by different degrees of multidisciplinarity considered in the simulations and, if so, to what extent. For this purpose, the gust interaction simulations were carried out in three different ways. The starting point for each type of simulation is the aircraft's trimmed aeroelastic equilibrium configuration associated with the respective flight conditions.

1. Pure CFD analysis: In this simulation, the aircraft is completely fixed in the initial position while being exposed to the gust. No flight dynamic or elastic degrees of freedom are considered.

2. Coupled CFD-FD analysis: The aircraft has only flight dynamic degrees of freedom. During gust encounter, the aircraft in trim configuration is allowed to undergo rigid body motions only.

3. Coupled CFD-FD-CSD analysis: The aircraft has flight dynamic and elastic degrees of freedom. The aircraft's reaction to the gust loads is a superposition of rigid body motions and elastic deformation.

DLR applied the Digital-X multidisciplinary process chain for the simulation of the CFD4Loads test cases, using TAU as CFD solver in fully turbulent RANS mode. For gust modeling, the existing TAU disturbance velocity approach was used [36], [37]. It considers the influence of the gust on the aircraft, but not the reaction of the aircraft on the gust. However, it has been shown that the disturbance velocity approach is sufficiently accurate if the ratio of the gust wavelength to the aircraft characteristic length exceeds the value of two [38]. This holds for all CFD4Loads gust cases investigated. "1-cos" gusts were

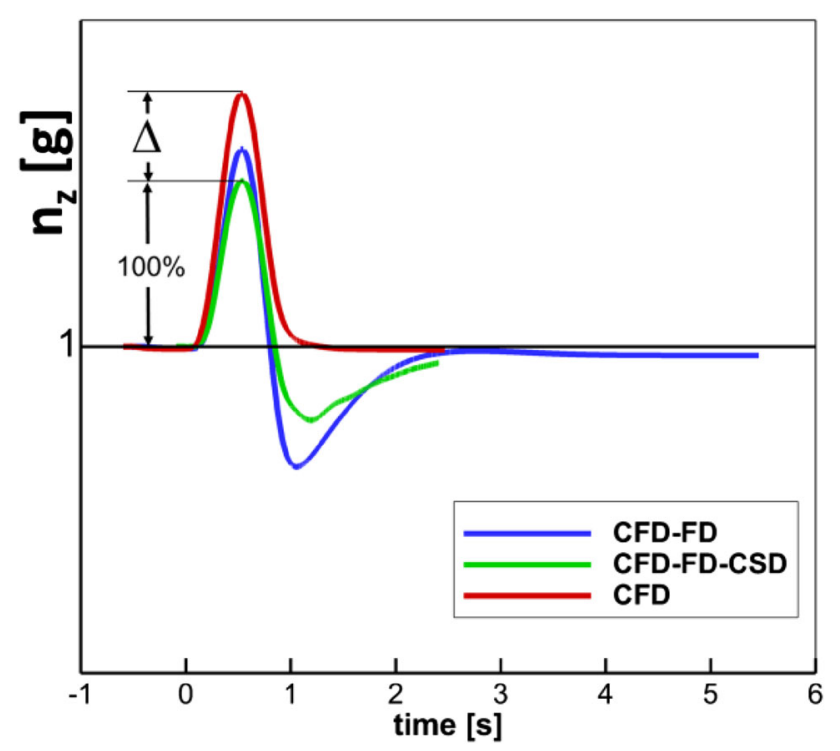

Fig. 24 Effect of multidisciplinarity on the time histories of the load factor $n_{\mathrm{z}}$ during a gust interaction case in transonic flow simulated according to the definitions in CS 25.341 (a) [39].

Figure 23 shows the trimmed aeroelastic equilibrium configuration for a subsonic case $(M=0.45$, $R e=70 \times 10^{6}, m=275 \mathrm{t}$ ). Here, the results of the CFDbased process and those of the conventional process which is based on linearized aerodynamics are in good agreement.

The gust interaction simulations were then carried out based on the trim configurations. Figure 24 shows the time histories of the predicted load factor $n_{\mathrm{z}}$ obtained for simulations with different degrees of multidisciplinarity $\left(n_{\mathrm{z}}\right.$ is measured at an aircraft fixed reference point). The time $t=0$ in the diagrams of Fig. 24 corresponds to the moment when the gust reaches the nose of the aircraft. In all simulations the time step was chosen so that the respective gust period was resolved with well over 100 time steps. Test simulations with close coupling did not show significantly different results compared with loose coupling. The latter was therefore used for all further simulations.

The investigation of various levels of multidisciplinarity in the simulations shows the expected consistent behavior. The highest load factors occur in the single-discipline analysis (only CFD), the lowest when considering the full scope of multidisciplinarity (coupled CFD-FM-CSM). The differences in the maximum load factor between the two extremes is about $\Delta=63 \%$.

Figure 25 shows the state of the aircraft in the transonic gust load case at the time of the peak load factor as predicted in Digital-X with CFD-FM-CSM coupling. The additional wing bending caused by the reaction to the gust load is clearly seen when compared to the shape of the trimmed aeroelastic equilibrium configuration.

As a result of the findings in the CFD4Loads initiative regarding the prediction of aircraft-gust interactions using the Digital-X process, the selective use of CFD in the context of load analysis has now been intensified by the industry partner.

\subsection{Quantifying uncertainties using numerical simulation}

Aerodynamic and aeroelastic input parameters (flow conditions, geometry, material quantities, etc.) are often subject to significant uncertainties, which are not usually considered in the established deterministic simulation methods. However, quantitative information on the effects of such uncertainties is desirable for assessing simulation results and ultimately necessary for virtual certification. The objective of this work package is to provide efficient methods and tools for quantifying output uncertainty using numerical simulation. 


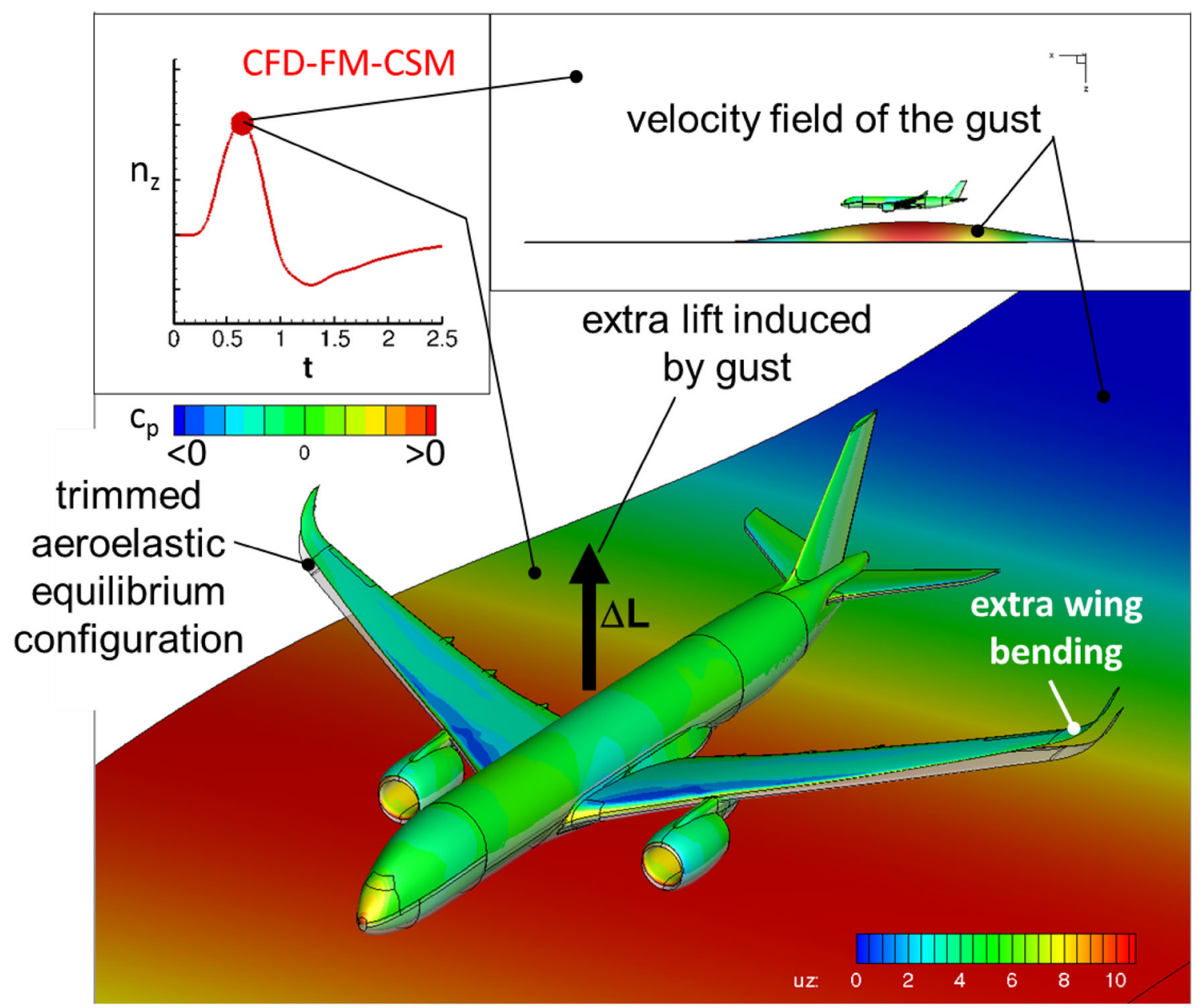

Fig. 25 Visualization of the gust response of the analyzed aircraft as predicted by the Digital-X process (CFD-FM-CSM simulation of the transonic gust test case)

\subsubsection{Non-intrusive method}

Based on input data considered as random fields, the random simulation results can be computed with intrusive or non-intrusive methods. The project Digital-X focuses on efficient non-intrusive methods which significantly reduce the computational cost compared to Monte Carlo methods, and make possible the treatment of distributed uncertainties such as those caused, for example, in geometry by manufacturing tolerances, or through wear, dirt or ice accumulation. Both gradient-assisted surrogate methods and reduced order models based on POD are employed.

Current research is concerned with parameterization of geometrical uncertainties for three-dimensional wings to model and analyze distributed uncertainties. One of the challenges was to implement the geometrical variations from a practical standpoint. To handle spatially correlated variation in the geometry an Eigen-decomposition of a very large, but rank deficient covariance matrix has to be made. In view of the computational complexity and memory required, methods have been developed for a coarse approximation to this matrix which maintains the geometric variance up to the machine precision. A parameterization in independent random variables is achieved by a subsequent Karhunen-Loève expansion of the random field based on the approximate matrix. After development the method was tested on a transport aircraft wing with 56,312 upper surface mesh points with uncertainties. In doing this particular assumptions were made about the correlation between any two mesh points such that the geometry variation is parameterized by only 600 instead of 56,312 random variables without any loss in the variance of the variation. The resulting eigenvalue problem could be solved very quickly and efficiently using a single core of a desktop computer. Figure 26 shows four examples of the random geometry parameterized with 600 variables.

\subsubsection{Methods for robust design}

Building on the methods for efficient quantification of distributed uncertainties that were developed in the work package above, stochastic optimization techniques have 


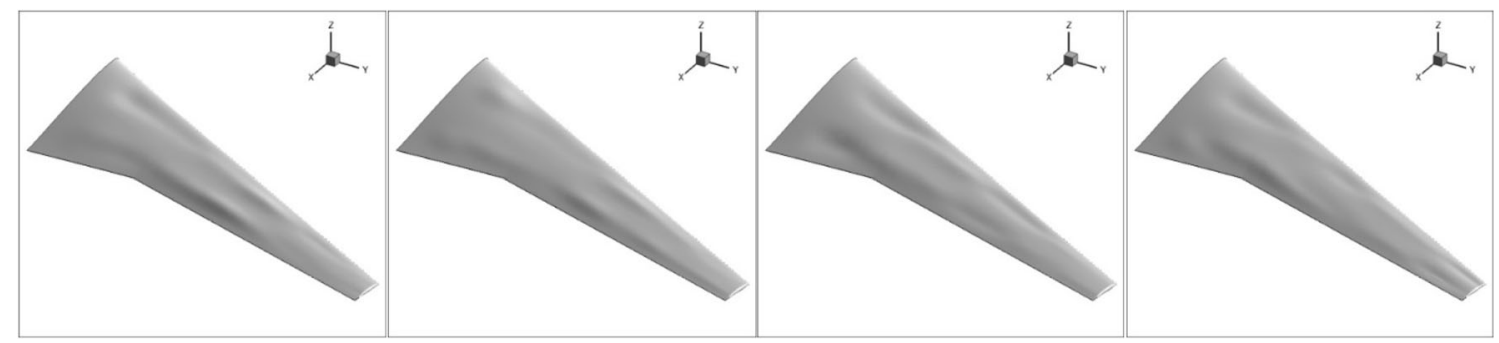

Fig. 26 Examples of random geometry deformations of a wing (600 variables)

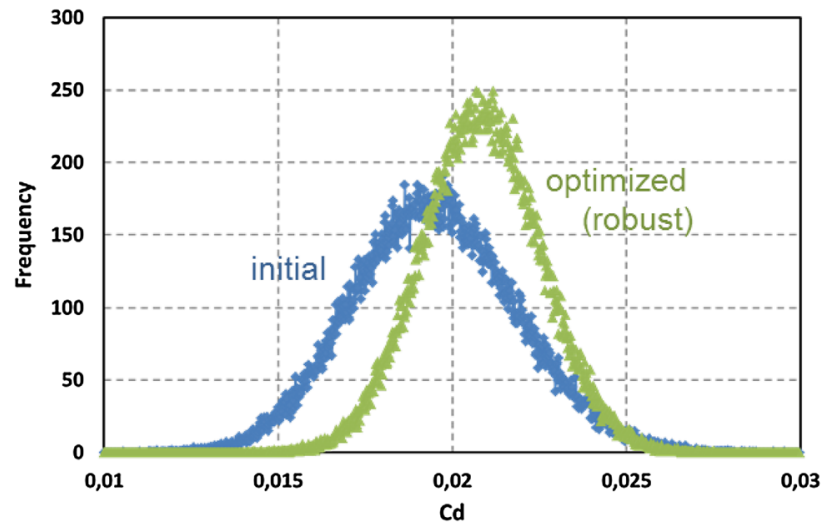

Fig. 27 Comparison of output PDFs: RANS-based robust design optimization (mean-risk approach) of Rae 2822 airfoil with uncertain geometry

been developed, which allow geometric variability in the design process to be considered and permit a robust design, i.e., a design that is less sensitive to small random perturbations.

The robust design formulation used is based on an expectation measure. The goal was to minimize the sum of the mean and standard deviation of the drag coefficient of the RAE 2822 airfoil for a given nominal lift coefficient. Here, both the flow conditions and the design parameters were considered uncertain. The nominal flow conditions were set to $M=0.734$ and $\alpha=2.79^{\circ}$. The standard deviations of these parameters were 0.005 and 0.1 , respectively. The geometric uncertainty was parameterized with the KLE, resulting in ten normally distributed uncertain geometry variables with a maximum standard deviation of 0.00125. The airfoil itself was parameterized with ten deterministic design variables. For each optimization step 100 samples were computed with the TAU code to determine the stochastic variation of the drag coefficient. They were used to construct a Kriging surrogate model for the uncertain objective function, which in turn was used to efficiently perform a full Monte Carlo simulation with Gaussian distributed variables to evaluate the statistics. As a result, the mean of the drag coefficient could be reduced by 60 drag counts while its standard deviation was reduced from 12.4 counts to five counts, requiring a total of about 500 iterations of the subplex optimization algorithm.

Other measures of robustness that have been considered so far include the worst-case risk measure and the meanrisk approach, which are both reliability-based robust design formulations. In the mean-risk approach, the idea is to maximize the probability that the drag coefficient is within a certain range around the mean value, requiring both the mean value and the probability density function (PDF) of the drag coefficient to evaluate the objective function. The result of this optimization in terms of the PDF of the initial and the optimized airfoil is shown in Fig. 27. The probability could be increased from 60.5 to $81.4 \%$ at the cost of a higher mean value. The influence of the different measures of robustness on the result of a $2 \mathrm{D}$ robust design optimization problem has also been investigated.

\subsection{Coupled CFD/CSM simulation of the trimmed helicopter}

The numerical simulation of the flow around helicopters is very expensive because it must be performed time-accurate and multidisciplinary. The calculations are complicated by a number of aerodynamic effects which occur during a revolution of the rotor, such as compression shocks, sheared boundary layers and flow separation. In addition, the blade tip vortex must be calculated very exactly to accurately predict blade/vortex interactions. Due to the strong aeroelastic deformation of the rotor blade, simulations must always be performed as a coupled fluid/structure computation. Furthermore, rotor trimming is required to set the required helicopter flight conditions.

In Digital-X, a multidisciplinary process chain for the complete trimmed helicopter including tail rotor under industrial conditions is developed, which builds on earlier work in the development and validation of simulation capabilities for isolated helicopter components. In contrast to previous work the unstructured TAU code is used because of the high geometric complexity and the requirement for automated mesh generation [40]. 


\subsubsection{Coupling methods}

To take into account the elasticity of the rotor blades and rotor trim, the CFD solver TAU was coupled with the comprehensive rotor code HOST (helicopter overall simulation tool [41]) of Airbus Helicopters in a previous research project. Here the RANS solver TAU computes the aerodynamic loads based on the blade deformations and control angles provided by HOST. The aerodynamic loads are then used to correct HOST's simplified aerodynamic model. The iterative process is carried out in the form of a so-called weak coupling. Whereas previously coupling was limited to single main rotor blades, in Digital-X the coupling chain is improved and extended with focus on applications to the complete helicopter. An important aspect is the development of a suitable protocol for transferring aerodynamic loads, determined by the TAU code on unstructured grids, to the beam-structure model of HOST. The transfer method was implemented such that in future more accurate finite element structural solvers can be used instead of beam models.

The design of the extended coupling environment allows different simulation tools to be used. Thus, the TAU code can be replaced by an alternative flow solver, or instead of the HOST code the DLR internally developed rotor simulation code S4 [42] can be used. As a step towards industrialization the coupling chain can be connected as TAU Python "plug-in" to the parallel simulation environment FlowSimulator.

\subsubsection{Chimera extensions}

For helicopter simulations the availability of the Chimera technique is essential because it allows a relative motion of the component meshes so that rotors, etc., can be simulated. In the Chimera technique the components of a configuration are first meshed independently. Subsequently the component meshes are combined into a mesh system, in which the meshes partially overlap each other. To ensure a valid solution, the data are transferred between the

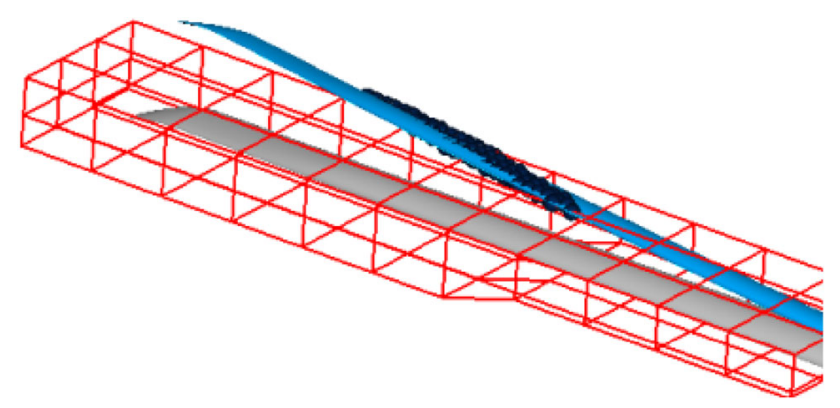

Fig. 28 Position of the deformed rotor blade (blue) relative to the original position (gray) and the hole definition geometry (red), GOAHEAD rotor overlapping mesh blocks by interpolation. Additionally the nodes of a component mesh which are inside the body of another mesh must be excluded from the calculation.

For the hole cutting procedure so-called "hole definition geometries" are used, which consist of simple geometric shapes. In the case of a rotor simulation with large blade deformations it is possible that a deformed blade protrudes from the originally specified hole cutting geometry during the computation (Fig. 28). This leads to an irregular data transfer and termination of the computation.

A possible solution would be the generation of a large overlap region for the blade mesh and background mesh as well as the definition of a sufficiently large hole geometry, so that the deformed blade does not leave the defined hole geometry during the simulation. However, this is not suitable in complex configurations as the proximity of the main and tail rotor to the fuselage does not allow large overlap regions.

An alternative approach is to consider the rotor blade deformation during the hole cutting procedure in determining the nodes to be eliminated. This can be achieved by deforming the hole definition geometries according to the blade deformation (Fig. 29). In this way narrow regions of grid overlap can be realized.

\subsubsection{First results from the coupled TAU/HOST simulations}

To validate the TAU/HOST coupling process chain, test data for cruise flight were selected from the GOAHEAD wind tunnel test campaign in the DNW/LLF [43]. The wind tunnel model (Fig. 30) consists of the ONERA 7AD main rotor, a BO105 tail rotor and a generic transport helicopter fuselage. In the first step the TAU/HOST simulations were performed for the main rotor [44]. In addition, numerical reference results are available from the validated FLOWer/HOST process chain. FLOWer is

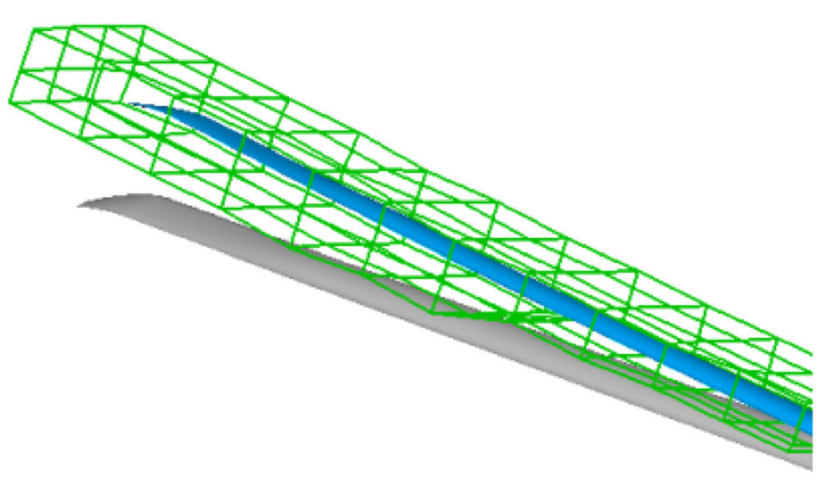

Fig. 29 Position of the deformed rotor blade (blue) relative to the original position (gray) and the deformed hole definition geometry (green), GOAHEAD rotor 


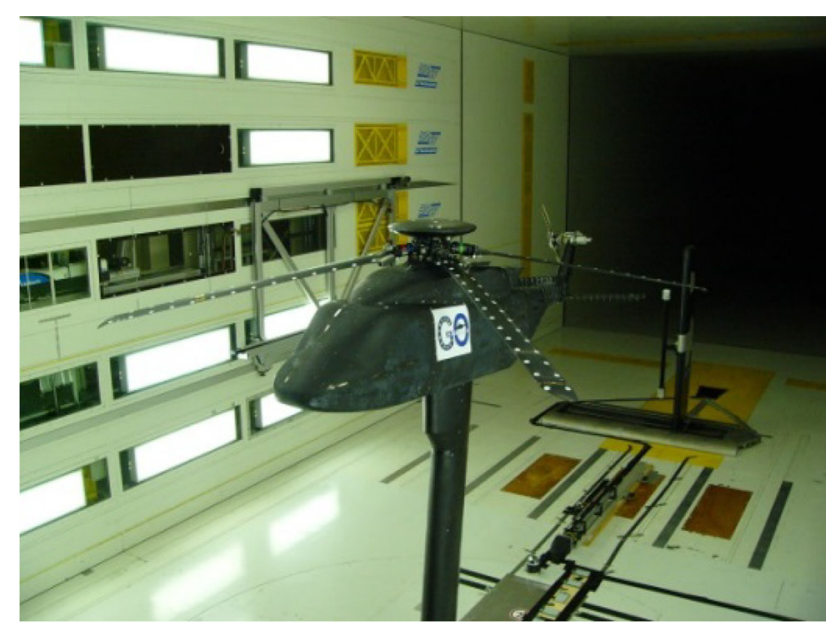

Fig. 30 Wind tunnel set-up in GOAHEAD

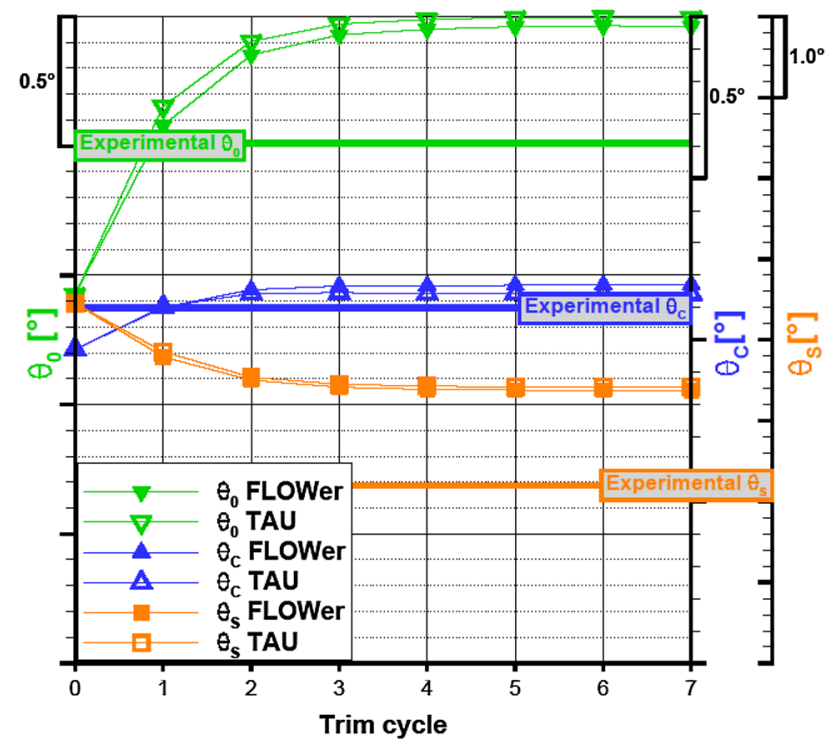

Fig. 31 Convergence behavior of the control angles, comparison between TAU/HOST, FLOWer/HOST and experimental data for the GOAHEAD main rotor

DLR's block-structured RANS solver. The TAU calculations were performed on the structured FLOWer grids, to exclude grid effects in the validation.

Figure 31 shows the convergence of the control angles. The first trim cycle was carried out after three rotor revolutions, with the following trim cycles performed after each further rotor rotation. The simulation was terminated after the incremental changes in all control angles were less than $0.001^{\circ}$.

The convergence behavior of the two simulations is very similar. Convergence was reached after seven trim iterations. The numerical results agree very well with each other. The deviations from the experimental results in the collective $\left(\theta_{0}\right)$ and lateral $\left(\theta_{\mathbf{C}}\right)$ control angles are relatively small under $0.5^{\circ}$. A larger deviation is observed only in the longitudinal control angle $\left(\theta_{\mathrm{S}}\right)$. This is mainly caused by the lack of interaction with the fuselage. It is concluded from the detailed comparison of the results that the TAU/ HOST coupling was successfully validated for the isolated 7AD main rotor. Future activities will focus on the simulation of the complete GOAHEAD configuration.

\subsubsection{Improvement in accuracy of blade/vortex interactions}

The accurate prediction of the blade/vortex interaction, which has a significant influence on aerodynamic and aeroacoustic loads, requires the correct transport of vortices through the flow field. The DLR TAU code uses a secondorder discretization of the RANS equations, so that simulations of the blade tip vortices and blade-vortex interactions require extremely fine computational grids due to the relatively high inherent numerical dissipation.

This restriction should be lifted in Digital-X through coupling the TAU code with a RANS higher order method. The fourth order FLOWer version (FLOWer-4, [45]) was chosen for this. A coupling method for two CFD codes was already developed within the DFG Research Unit FOR1066, [46, 47]. This method is based on the Chimera technique and is implemented as a separate coupling module. The flow solvers require only an appropriate communication interface for data transfer and a suitable boundary condition for defining flow data on the coupling surface. This approach allows a flexible coupling between different flow solvers. In the context of Digital-X the necessary improvements and extensions to TAU and FLOWer-4 are currently being carried out so that specific helicopter applications can be performed with the coupled procedure. The communication interface in FLOWer-4 has already been implemented and successfully tested with a two-dimensional test case. Currently the coupling is being extended to allow for relative motion of component grids and the predictive accuracy of the coupled approach is tested on an isolated rotor.

\subsection{Automatic process chain}

Complex multidisciplinary simulations and optimizations require automated process chains. The main points here are the flexible yet efficient connection of different software components, the application of massively parallel supercomputers, as well as support in the use of automated processes. Thus, in Digital-X specific developments are being undertaken in the fields of a parallel multidisciplinary simulation environment and a workflow management system. 


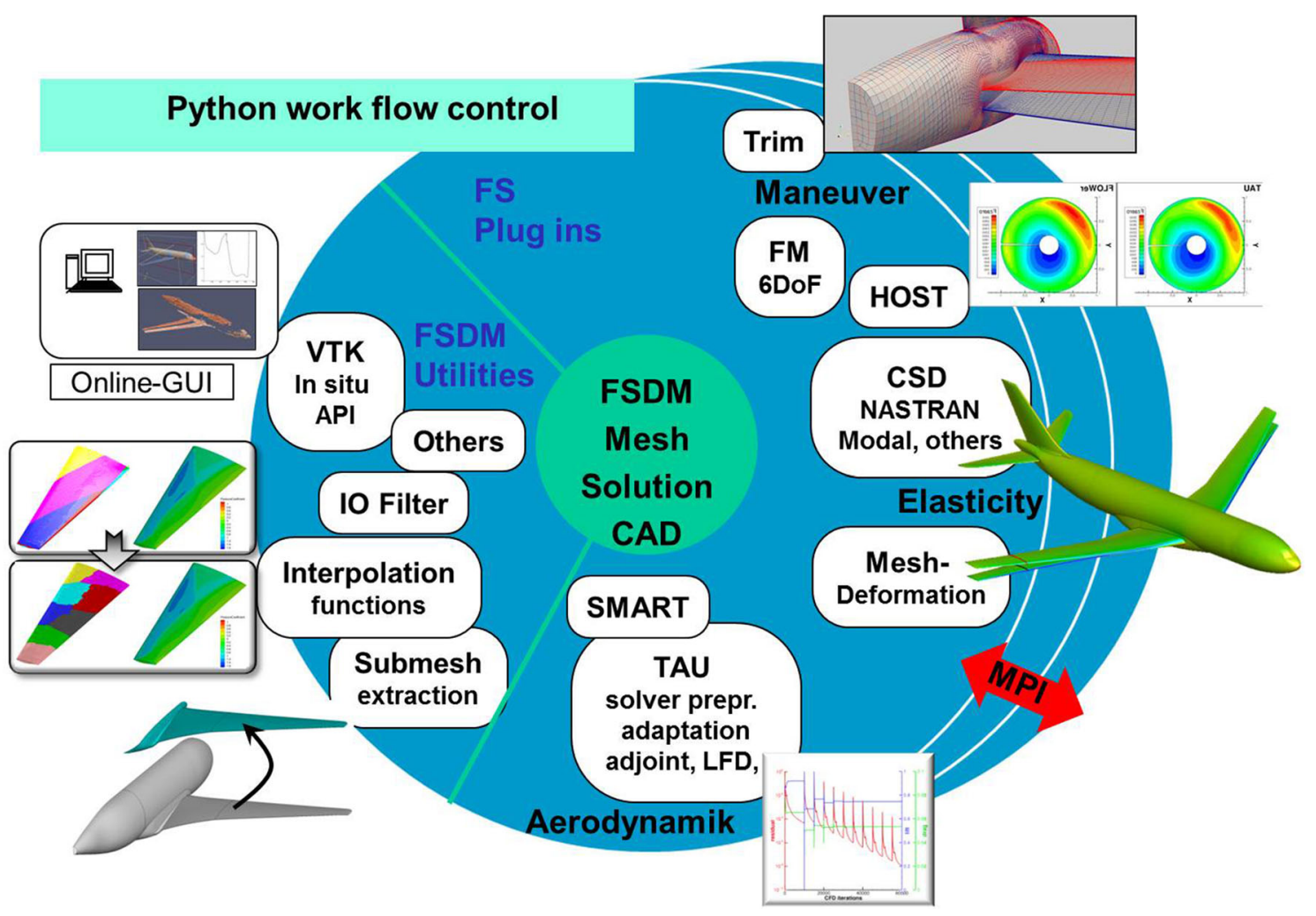

Fig. 32 Application scenarios for the FlowSimulator

\subsubsection{FlowSimulator environment}

The simulation environment in Digital- $\mathrm{X}$ is based on the FlowSimulator software which was developed by Airbus and DLR in cooperation with other European research partners [30]. This software manages in parallel CFD data (meshes, solvers, CAD and meta-data), so that various programs (e.g., CFD and CSM software at different levels, and pre- and post-processing) can be coupled without filebased data exchange (file-IO).

First extensive developments for multidisciplinary simulation were carried out in the framework of the $C^{2} A^{2} S^{2} E$ project [35]. FlowSimulator allows coupling of different programs within a so-called MPI communicator, in which the parallel distributed programs control data exchange via the shared memory of the individual MPI processes. The process chain is defined by a Python script which controls the sequence of programs per MPI process. The aim of the work in Digital-X is to integrate the newly developed capabilities and to provide necessary scripts for the planned multidisciplinary analysis and optimization scenarios. The high parallel efficiency of the computation chains plays a crucial role. Figure 32 gives a schematic overview of the essential applications of FlowSimulator.

\subsubsection{RCE Workflow management system}

For setting up and controlling the computational workflow the management system RCE developed within DLR [31] is used. The main objective here is to further develop RCE for complex multidisciplinary simulations based on highly accurate computations (see also Sect. 3.3). The management task includes control of one or more completed remote simulations that take place simultaneously on one or more computer clusters. This involves job start and runtime control with data transfer from input and output files over the network between the workstation and target computer, taking into account that queuing systems must usually be addressed there. Furthermore, the optimizer workflow component should be extended to Digital-X requirements.

Figure 33 shows the RCE workflow of the detailed level process of Fig. 9, which was used to produce the results shown in section Fig. 14. It contains the components which represent the disciplinary tools (CFD, structural sizing, etc.) as well as service components, which adapt the inputs and outputs between different tools and establish iterative loops. To run all the tools in this particular workflow, RCE orchestrated the use of an HPC cluster, two Linux-based 
Fig. 33 RCE workflow implementing a high-fidelity based aero-structure optimization process

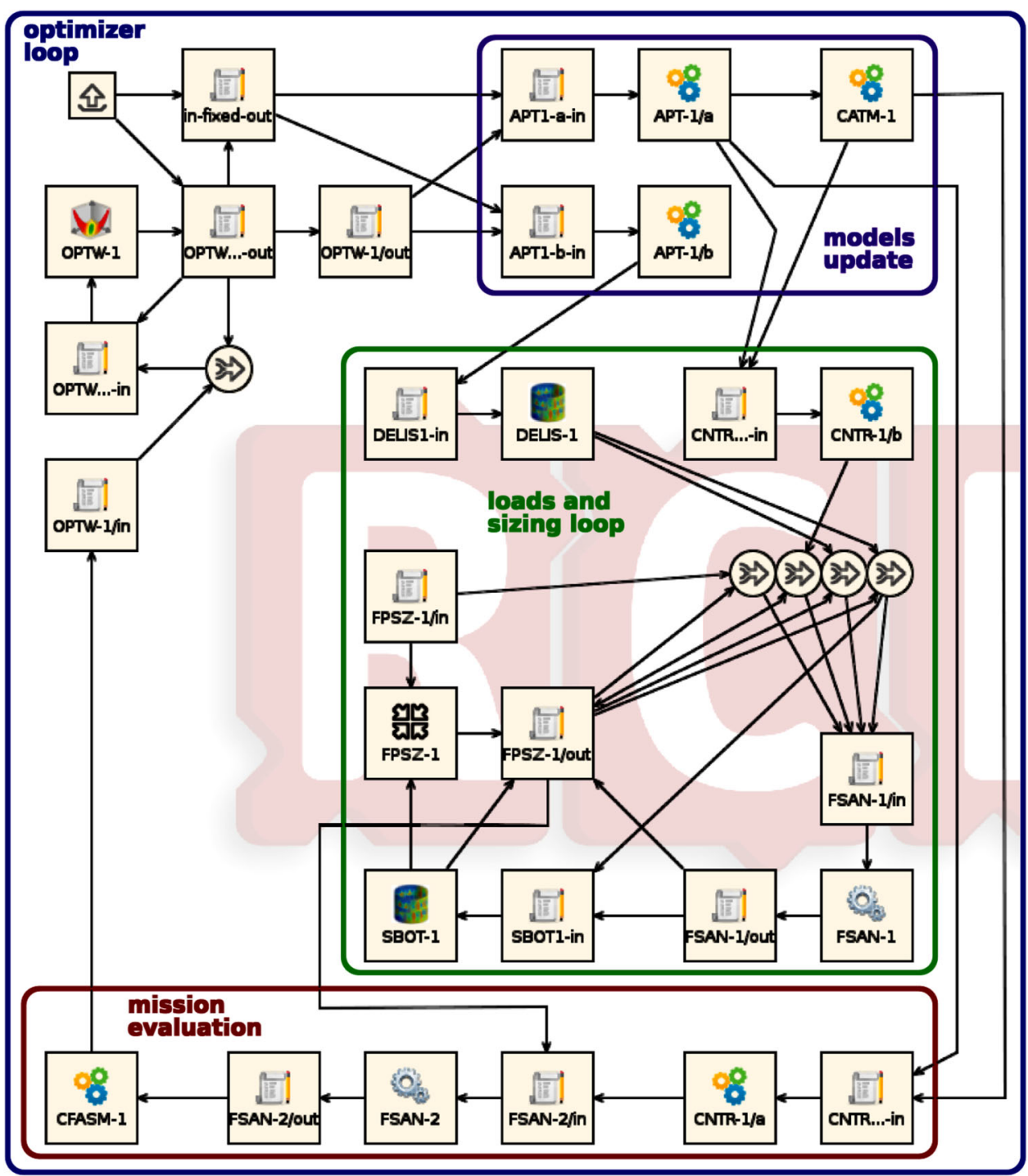

workstations, and two Windows-based workstations, spread over two different DLR institutes.

\section{Summary and next steps}

At DLR, a multidisciplinary research project has been setup. It represents a first significant step towards realizing DLR's vision of the digital aircraft and virtual flight testing. Dedicated developments in disciplinary and multidisciplinary simulation methods are being addressed with a focus on multidisciplinary design and analysis of aircraft and helicopters.

The challenges in terms of physical modeling across the flight envelope require further improvements and enhancements of the DLR's well-established flow solver TAU. In Digital-X, the efficiency, robustness, reliability and level of automation of the TAU code is significantly improved and its range of applications is expanded. Given the technology development of high-performance computers, most CFD solvers used today have reached the limits of scalability when it comes to parallelization. Therefore, the design and implementation of a next generation flow solver are a key objective of the project. Besides providing the best possible utilization of future HPC systems, the new CFD code consolidates existing algorithms and incorporates innovative simulation approaches. The development of the next generation CFD solver is to be regarded as a future investment of DLR needed to address challenges in numerical simulation beyond the current project. The software specification has been completed and a prototype has already been implemented and evaluated. A first release for selected target applications is scheduled for the end of the project.

A multi-level/multi-fidelity MDO concept and process architecture have been defined and implemented based on 
improved disciplinary tools and disciplinary models that are generated automatically based on a common parametric description of the XRF-1 reference long-range transport aircraft configuration. An aero-structural optimization of a simplified XRF-1 with a metallic structure has been performed based on high-fidelity models and tools for a simplified mission and selected load cases, reducing the block fuel mass by $3.6 \%$. Next, the full multi-level MDO chain will be exercised on the full XRF-1 with additional design parameters and constraints and for a more complex mission. Further design load cases will also to be considered. Correction techniques and the developed reduced order models will be applied to improve efficiency and accuracy in the loads process. Finally, the entire MDO process chain is to be converted to a gradient-based optimization chain.

The results of the high-fidelity based multidisciplinary simulations obtained so far have demonstrated the general feasibility of such an advanced and ambitious venture. Planned simulations of the free-flying aircraft will include a flight control system to predict structural loads even more realistically. A series of steady and unsteady maneuvers including gust and wake vortex encounter scenarios will be simulated to further demonstrate the power of CFD-based aircraft analysis.

Acknowledgments The Digital-X project is a large, multidisciplinary project with many dedicated colleagues and external partners. The authors would like to thank all who were involved in the discussions and research activities, particularly those colleagues whose contributions were not explicitly mentioned in this overview article because of the size limitations.

Open Access This article is distributed under the terms of the Creative Commons Attribution 4.0 International License (http://crea tivecommons.org/licenses/by/4.0/), which permits unrestricted use, distribution, and reproduction in any medium, provided you give appropriate credit to the original author(s) and the source, provide a link to the Creative Commons license, and indicate if changes were made.

\section{References}

1. Flightpath 2050-Europe's vision for aviation, report of the high level group on aviation research. http://acare4europe.com, ISBN 978-92-79-19724-6, European union (2011)

2. Slotnick, J., Khodadoust, A., Alonso, J., Damofal, D., Gropp, W., Lurie, E., Mavriplis, D.: CFD vision 2030 study: a path to revolutionary computational aerosciences. http://ntrs.nasa.gov, NASA/ CR-2014-218178, NASA Center for AeroSpace Information, 7115 Standard Drive, Hanover, MD 21076-1320, US (2014)

3. Dean, J.P., Clifton, J.D., Bodkin, D.J., Ratcliff, J.: High resolution CFD simulations of maneuvering aircraft using the CREATE-AV/Kestrel solver. In: AIAA 2011-1109, 49th AIAA Aerospace Sciences Meeting, Orlando, Florida, 4-7 Jan 2011

4. Wissink, A. et al.: Capability enhancements in version 3 of the helios high-fidelity rotorcraft simulation code. In: 50th AIAA Aerospace Science Meeting, Nashville, Tennessee, 9-12 Jan 2012
5. Kenway, G.K.W., Martins, J.R.R.A.: Multipoint high-fidelity aero-structural optimization of a transport aircraft configuration. J. Aircr. 51, 1 (2014)

6. Schwamborn, D., Gerhold, T. Heinrich, R.: The DLR TAU code: recent applications in research and industry. In: Proceedings of European Conference on Computational Fluid Dynamics, ECCOMAS CDF 2006, Delft, The Netherland (2006)

7. Eisfeld, B.: Numerical simulation of aerodynamic problems with the SSG/LRR- $\omega$ Reynolds stress turbulence model using the unstructured TAU code, In: Tropea, C., Jakirlic, S., Heinemann, H.-J., Henke, R., Hönlinger, H. (eds.), Contributions to the 15th STAB/DGLR Symposium, Darmstadt, Germany 2006, Notes on Numerical Fluid Mechanics and Multidisciplinary Design, vol. 96, pp. 356-363, Springer, Berlin Heidelberg (2008)

8. Cecora, R.-D., Eisfeld, B., Probst, A., Crippa, S., Radespiel, R.: Differential reynolds stress modeling for aeronautics. In: AIAAPaper 2012-0465 (2012)

9. Eisfeld, B., Probst, A.: Industrial application of differential reynolds stress models, DLR-IB 2012, ISSN 16147790, 2012

10. Togiti, V., Eisfeld, B., Brodersen, O.: Turbulence model study for the flow around the NASA common research model. J. Aircr. 51(4), 1331-1343 (2014)

11. Probst, A., Reuß, S.: Scale-resolving simulations of wall-bounded flows with an unstructured compressible flow solver. In: 5th Symposium on Hybrid RANS-LES Methods, TEXAS A\&M University, College Station, Houston, USA, 19-21 Mar 2014

12. Grabe, C., Krumbein, A.: Correlation-based transition transport modeling for three-dimensional aerodynamic configurations. J. Aircr. 2013(50), 1533-1539 (2013)

13. Grabe, C., Krumbein, A.: Extension of the $\gamma$-Re $\theta t$ model for prediction of crossflow transition. In: AIAA 2014-1269, 52nd Aerospace Sciences Meeting (2014)

14. Langer, S., Schöppe, A. Kroll, N.: Investigation and comparison of implicit smoothers applied in agglomeration multigrid in the framework of the DLR TAU code. AIAA J. 53(8), 2080-2096 (2015)

15. Allmaras, et al.: Modifications and clarifications for the implementation of the Spalart-Allmaras turbulence MODEL, ICCFD71992. Big Island, Hawaai (2012)

16. Langer, S.: Agglomeration multigrid methods with implicit Runge-Kutta smoothers applied to aerodynamic simulations on unstructured grids. J. Comput. Phys. 277, 72-100 (2014)

17. Jägersküpper, J., Simmendinger, CH.: A novel shared-memory thread-pool implementation for hybrid parallel CFD solvers. In: Proceedings Euro-Par 2011, Lecture Notes in Computer Science, Band 6853, Springer, S. 182-193 (2011)

18. Tenenbaum, J.B., De Silva, V., Langford, J.C.: A global geometric framework for nonlinear dimensionality reduction. Science 290(5500), 2319-2323 (2000)

19. Holmes, P., Lumley, J., Berkooz, G.: Turbulence, Coherent Structures, Dynamical Systems and Symmetry. Cambridge University Press, New York (1996)

20. Bui-Thanh, T., Damodaran, M., Willcox, K.: Proper orthogonal decomposition extensions for parametric applications in compressible aerodynamics. In: AIAA 2003-4213, 21st AIAA Applied Aerodynamics Conference, Orlando, Florida, June 2003

21. Franz, T., Zimmermann, R., Görtz, S., Karcher, N.: Interpolationbased reduced order modelling for steady transonic flows via manifold learning, in special issue: reduced order modelling: the road towards real-time simulation of complex physics. Int $\mathbf{J}$ Comput Fluid Dynam 28(3-4), 106-121 (2014)

22. Zwaan, R.J.: LANN Wing. Pitching oscillation, compendium of unsteady aerodynamic measurements, Addendum No. 1, AGARD-R-702 (1985)

23. Thormann, R., Dimitrov, D.: Correction of aerodynamic influence matrices for transonic flow. CEAS Aeronaut J 5, 435-446 (2014) 
24. Parameswaran, V., Baeder, J.D.: Indicial aerodynamics in compressible flow-direct computational fluid dynamic calculations. J. Aircr. 34(1), 131-133 (1997)

25. Thormann, R., Widhalm, M.: Linear-frequency-domain predictions of dynamic-response data for viscous transonic flows. AIAA J. 51(11), 2540-2557 (2013)

26. Dietz, G., Schewe, G., Kiessling, F., Sinapius, M.: Limit-cycleoscillation experiments at a transport aircraft wing model. International Forum of Aeroelasticity and Structure Dynamics (IFASD), Amsterdam, The Netherlands (2003)

27. Liersch, C.M., Hepperle, M.: A distributed toolbox for multidisciplinary preliminary aircraft design. CEAS Aeronaut. J. 2(1-4), 57-68 (2011)

28. Nagel, B., Zill, T., Moerland, E., Böhnke, D.: Virtual aircraft multidisciplinary analysis and design processes-lessons learned from the collaborative design project VAMP. In: Proceedings 4th CEAS Air and Space Conference, Linköping, Schweden (2013)

29. Ronzheimer, A., Natterer, F.J., Brezillon, J.: Aircraft wing optimization using high fidelity closely coupled CFD and CSM methods. In: AIAA Paper 2010-9078, 13th AIAA/ISSMO Multidisciplinary Analysis Optimization Conference, Fort Worth, Texas, Fort Worth, Texas (2010)

30. Meinel, M., Einarsson, G.O.: The FlowSimulator framework for massively parallel CFD applications, PARA 2010. PARA2010, 6-9 June 2010, Reykjavik, Island (2010)

31. Seider, D., Zur, S., Flink, J., Mischke, R., Seebach, O.: RCEDistributed, workflow-driven integration environment, EclipseCon Europe 2013, 29-31 Oct 2013, Ludwigshafen (2013)

32. Rizzi, A., Zhang, M., Nagel, B., Böhnke, D., Saquet P.: Towards a unified framework using CPACS for geometry management in aircraft design. In: AIAA Paper 2012-0549, AIAA Aerospace Sciences Meeting, Nashville, USA (2012)

33. Liepelt, R.; Chiozzotto, G.P., Schmidt, H.: Variable fidelity loads process in a multidisciplinary aircraft design environment. In: 4th CEAS Air and Space Conference (2013)

34. Freund, S., Heinecke, F., Führer, T., Willberg, C.: Parametric model generation and sizing of lightweight structures for a multidisciplinary design process. In: NAFEMS DACH-Tagung, 20-21 May 2014, Bamberg Germany (2014)

35. Kroll, N., Heinrich, R., Görtz, S., Krumbein, A., Gerhold, T.H.: Abschlussbericht des Projektes $C^{2} A^{2} S^{2} E$, DLR-IB 124-2014/3, ISSN 1614-7790 (2014)

36. Heinrich, R., Reimer, L., Michler, A.: Multidisciplinary simulation of maneuvering aircraft interacting with atmospheric effects using the DLR TAU code, RTO AVT-189 Specialists Meeting on Assessment of Stability and Control Prediction Methods for Air and Sea Vehicles, 12-14 Oct, Portsdown West, UK (2011)

37. Heinrich, R.: Simulation of Interaction of Aircraft and Gust Using the TAU code. In: Dillmann, A. et al. (eds.), New Results in Numerical and Experimental Fluid Mechanics IX, Notes on Numerical Fluid Mechanics and Multidisciplinary Design, Springer, Band 124, S. 503-511 (2014)

38. Heinrich, R., Reimer, L.: Comparison of different approaches for gust modeling in the CFD Code TAU, international forum on aeroelasticity and structural dynamics, 24-27 June, Bristol, UK (2013)

39. European Aviation Safety Agency (EASA), Certification Specifications for Large Aeroplanes CS-25. Volume Subpart CStructure (2010)

40. Khier, W., Dietz, M., Schwarz, T., Wagner, S.: Trimmed CFD Simulation of a Complete Helicopter Configuration, 33rd European Rotorcraft Forum. Kazan, Russia (2007)

41. Benoit, B., Dequin, A.-M., Kampa, K. W., Gruenhagen, P.-M., Basset, P, B.: HOST, A General Helicopter Simulation Tool for Germany and France. In: 56th Annual Forum of the American Helicopter Society Virginia Beach, USA (2000)

42. Van der Wall, B.G., Yin, J.: DLR's S4 Rotor code validation with HART II data: the baseline case, 1st international forum on rotorcraft multidiscipinary technology, Seoul, Korea, 15-17 Oct 2007

43. Pahlke, K.: The GOAHEAD project. In: Proceedings of the 33rd European Rotorcraft Forum, Kazaan, Russia (2007)

44. Wendisch, J.-H., Raddatz, J.: Validation of an unstructured CFD solver for complete helicopter configurations with loose CSDtrim coupling, 40th European Rotorcraft Forum, Southampton, UK, 2.-5. September 2014

45. Enk, S.: Ein Verfahren höherer Ordnung in FLOWer für LES, DLR-IB-124-2007/8 (2008)

46. Spiering, F.: Coupling of TAU and TRACE for parallel accurate flow simulations. In: International Symposium on Simulation of Wing and Nacelle Stall, Braunschweig, Germany (2012)

47. Spiering, F., Kelleners, P.: Coupling of flow solvers with variable accuracy of spatial discretization, new results in numerical and experimental fluid mechanics IX. In: Contributions to the 18th STAB/DGLR Symposium, Stuttgart, Germany, pp. 415-423, Springer, ISBN 978-3-319-03157-6 (2012) 